\title{
Implication of scaling hierarchy associated with nonequilibrium: field and particulate
}

\author{
G.C. Sih ${ }^{a, b, c, *}$ \\ ${ }^{a}$ Department of Mechanical Engineering and Mechanics, Lehigh University, Bethlehem, PA 18015, USA \\ b Institute of Mechanics, Chinese Academy of Sciences, Beijing 100080, China \\ ${ }^{\mathrm{c}}$ Institute of Engineering Mechanics, Hebei University of Technology, Tianjin 300130, China
}

\begin{abstract}
The advent of nanotechnology has necessitated a better understanding of how material microstructure changes at the atomic level would affect the macroscopic properties that control the performance. Such a challenge has uncovered many phenomena that were not previously understood and taken for granted. Among them are the basic foundation of dislocation theories which are now known to be inadequate. Simplifying assumptions invoked at the macroscale may not be applicable at the micro- and/or nanoscale. There are implications of scaling hierrachy associated with inhomegeneity and nonequilibrium of physical systems. What is taken to be homogeneous and equilibrium at the macroscale may not be so when the physical size of the material is reduced to microns. These fundamental issues cannot be dispensed at will for the sake of convenience because they could alter the outcome of predictions. Even more unsatisfying is the lack of consistency in modeling physical systems. This could translate to the inability for identifying the relevant manufacturing parameters and rendering the end product unpractical because of high cost. Advanced composite and ceramic materials are cases in point.

Discussed are potential pitfalls for applying models at both the atomic and continuum levels. No encouragement is made to unravel the truth of nature. Let it be partiuclates, a smooth continuum or a combination of both. The present trend of development in scaling tends to seek for different characteristic lengths of material microstructures with or without the influence of time effects. Much will be learned from atomistic simulation models to show how results could differ as boundary conditions and scales are changed. Quantum mechanics, continuum and cosmological models provide evidence that no general approach is in sight. Of immediate interest is perhaps the establishment of greater precision in terminology so as to better communicate results involving multiscale physical events. () 2001 Published by Elsevier Science Ltd.
\end{abstract}

... the continuous field thus appeared side by side with the material point as the representative of physical reality. This dualism remains even today, disturbing as it may be to every orderly mind.

Albert Einstein

\section{Introduction}

\footnotetext{
${ }^{*}$ Present address: Xi'an Jiatong University, School of Advanced Science and Technology, Xi'an Shaanxi 710049, China. Fax: 86-29-2668028.

E-mail address: gcs@xjtu.edu.cn (G.C. Sih).
}

Nanosize products could lead the challenge in science and technology of the 21st century since they can have far reaching consequences on the ways with which world economy would evolve. US 
government research support and venture capital funding have already pumped sizable investments into nanotechnology that will soon reach in the billions. In the year 2000, \$270 millions were spent by the US government in nanotechnology; this amount will increase to about half-a-billion dollars in the year 2001. Close to $\$ 72$ millions of dollars have been earmarked for nanotechnology by venture capital films such as Carbon Nanotechnology in material science, Quantum Dot and C Sixty in medicine and Siwave and Arryx in optical switching [1]. The manufacturing of nanotubes is already well known with activities spearheaded by Mitsubishi, Materials and Electrochemical Research, Research Corporation Technologies and other equally bonafide companies. These tubes are extremely tiny and equivalent to only about $1 / 80,000$ the diameter of a human hair. However, they are stronger than any known fibers today and would be ideal in composite materials. Possibly, they could replace carbon black in automobile tires to increase the life of the tread. Nanomaterial research is also expected to make big breakthroughs in other fields such as microelectronics and nanoscale medicine.

The road to success, however, is not altogether straightforward. There are stumbling blocks to overcome as physics and chemistry have to converge on manufacturing. Massive and complex systems have to be grown atom by atom to produce macroscale materials. Replication of trillions of copies of atoms requires a knowledge of scaling hierarchy and the ways to characterize materials properties at the nano-, micro-, meso- and macroscale. Engineers would have to dig deep into the fundamentals of physics hoping that ideas and methodologies of yesteryears could be borrowed to unravel their present problems. Such an undertaking would likely to be more difficult than the development of a new set of disciplines to handle nanotechnology. The margin of errors for estimating events at the cosmoscale differ drastically from those at the nanoscale. The same applies to nuclear energy for a blast in contrast to thermal energy allowed for a computer chip. Successful application of nanotechnology will not be made possible without a comprehensive understanding of the fundamentals of physics and chemistry.
The material engineers and scientists know well that the strength of idealized solids with orderly crystal lattice could differ significantly from that of polycrystals containing grain boundaries and defects by three orders of magnitude [2] or more as a rule. It required more than a century to learn how alloy structural steel could be used effectively in ordinary and hostile environments. Now that the characteristic length of the material internal structure has been reduced from $10^{-5}$ to $10^{-9} \mathrm{~m}$ (nanometer), a new set of learning experience would have to be gained. Handbook test data enforced by ASTM for specimens centimeters in size would no longer hold for specimens microns in size. The very concept of test standardization may be replaced by controlled manufacturing. Stresses in micron size specimen increase three orders of magnitude from MPa to GPa [3]. Does this mean that small-scale materials are stronger and less likely to break? Perhaps, the interpretation of strength based on stress is no longer valid at the nanoscale. Also, dislocations [4] may not be representative of defects that occurs in nanomaterials where subatomic imperfections would take precedence. Energy dissipation from heat is known to rise appreciably when a square centimeter of surface is reduced to a square micrometer. Heat flux in computer chips has been estimated to be $10^{5}-10^{6} \mathrm{~W} / \mathrm{m}^{2}$ at $300 \mathrm{~K}$. This is comparable to the heat flux range experienced by the reentry vehicle from earth orbit where the temperature is five times higher. If the trend in size reduction continues to the molecular scale, nanocomputers would give off as much heat per unit volume as gunpowder. Integrated circuit design could thus be limited in density by the amount of heat they dissipate. These forecasts may or may not be true since little is known on size scaling on mechanical systems. What is known today is that the presentday designers tend to underestimate the damping of miniaturized machines by a wide margin.

A resurgent of interest has occurred in largescale atomistic computations [5-9]. One of the objectives is to generate more detailed information about the movements of atoms and to explore the behaviors of solids due to change in spatial size scale. Results for yield strength of different size blocks made of nickel, gold and copper subjected 
to shear have been obtained using the embedded atom method $[8,9]$. Among the parameters varied are the boundary temperatures ( 300 and $500 \mathrm{~K}$ ), applied strain rate $\left(10^{7}-10^{12} / \mathrm{s}\right)$ and specimen size $\left(10^{4}-10^{6}\right.$ atoms or 2 to $\left.300 \mu \mathrm{m}\right)$. The specimen volume to surface ratio $V / A$ was used as a length parameter. It correlated well with the yield strength data for $V / A$ covering size orders of magnitude from $10^{-10}$ to $10^{-4} \mathrm{~m}$.

Whether scaling material behavioral parameters is a valid concept deserves scrutiny if the procedure is to cover a wide range from the nano- to the macroscale where the representation of nature as particulates or field becomes an issue. There is a good reason to raise this inquiry because microscopic entities do not have properties even remotely similar to those of macroscopic bodies. Although rules are necessary for establishing material behavior in an orderly sequence, it is not sufficient just to monitor the change in the morphology of the microstructures, either numerically or experimentally. It may just turn out that the complexity of nature defies scaling of any kind in general.

Other atomistic simulation methods consist of energy minimization [5] for systems at $0 \mathrm{~K}$; molecular dynamics [6] for including thermal vibrational effect of atoms; lattice Monte Carlo [7] for considering events where atom motion takes place from one lattice site to another; embedded atom method [8,9] mentioned earlier for incorporating many-body atomic potentials; and others that will sure be forthcoming. These large-scale computational schemes utilize hundred thousands and more atoms with time-steps smaller than the atomic vibrational frequencies of $10^{-13} \mathrm{~s}$. Unvalidated assumptions, speculations and empirical data are widespread in these programs. Frequently used are the electron wave functions for determining the forces acting on individual atoms by assuming that the corpuscular and continuum view of nature may be used interchangeably. Vibrational principles are expedient for optimizing conditions mathematically. There is no assurance that atoms in a real environment would adopt the pattern according to minimum energy of the system. Empirical atomic bonding potentials do not include the environmental dependence of bonding while the commonly used Lennard-Jones pair potential leaves out the effect of many-body interaction. Granted that the truth of nature may never be known, it would be more satisfying if more efforts can be made to determine the deviations of physical models from reality in a consistent manner. Oversimplification of physics is no trade-off for complexities in numerical computations.

Until macroscopic materials could be fabricated atom by atom with controllable and determinable properties at various scale levels, analytical models should be developed to quantify the combined influence of strain rate, temperature and specimen size, not to mention the evolution of imperfections and defects in the crystal lattice. Their existence should not be regarded as incidental but fundamental. Motion of atoms through crystals could not have occurred unless at least one of them was somehow displaced from its normal position in the structure. Such a displacement has been regarded as imperfection at the atomic scale. It is not unthinkable that similar events could be found at the subatomic scale. Imperfections or defects have contributed to possible explanation for a paradox related to superconductivity that were announced by IBM scientists in Zurich (1986). What seems baffling at the time was that "... a perfect version of the crystal in which every cell is identical to every other refuses to conduct any electricity". The then AT\&T Bell Laboratories at Holmdel, NJ observed that atomic planes are pulled out of place due to missing oxygen atoms and presence of yttrium atoms...". These defects themselves might be directly responsible for the superconductivity. They resemble earthquake faults in the otherwise orderly crystals and provide a way of creating an unusual interaction between electron flow and crystal vibration, an interaction that superconductivity seems to require". Another possible explanation was based on the concept of dislocation free zones ahead of crack-like fault lines [10]. These zones corresponding to local cooling were predicted analytically $[11,12]$. They suppress energy dissipation and hence dislocation emission. Dislocation free crack tip zones have been observed in single crystals of pure stainless steel, copper and aluminum in tensions [13]. Could it be the emission of dislocations from a multitude of 
fault lines that contribute to the superior mechanical and/or electrical performance?

Size reduction tends to reveal the corpuscular character of nature where atoms move continuously. They appear to vibrate about its equilibrium position. A motionless state is assumed to prevail at zero absolute temperature when thermal fluctuation ceases to exist. Short of an absolute base of reference, all descriptions would have to be relative from one scale to another. Macroevents are small on the cosmoscale but large on the nanoscale. Whether a system of particulates would converge to a smooth field remain as a conjecture. As long as the concept serves a useful purpose, it will continue to play a role in the development of science although the coexistence of nature as particulates and continuum will be argued.

Advancement in science and technology does not depend on learning the truth; it relies on the repeatability and consistent use of data. Shortcomings in the basic approaches, however, should be recognized and minimized for otherwise advancement would be short-lived. The discussions offered in the following are intended to encourage refinements and improvements toward the development of nanotechnology.

Limitation of quantum mechanics. Quantum electrodynamics has not been successful in attempts to reconcile the contrast between solid particles and the space surrounding them. The supposition is that particles are merely local condensations of the field. The quantum field theory relies on the interaction of forces between the particles. Any commitment made on particle size, however, implies that there exists even smaller particles. This process of searching for the smallest particle is obviously unsatisfying. Quantum mechanics, therefore, has not resolved the contradictory concepts of the continuum versus the granular structure. It merely suppresses the assessment to another lower scale or the quantum level.

Einstein was skeptical of the coexistence of field and particulates. His postulate on the particlewave model to justify the existence of phonons in electric insulators should not be regarded as particle-continuum duality. Wave and continuum are not synonymous in the context of this discussion.
"Particle wave" is a term used to describe quantum behavior of atomic objects. No intention is made to imply that nature is either particulates or continuum. It may be neither. Planck's length $4 \times 10^{-33} \mathrm{~cm}$, time $10^{-42} \mathrm{~s}$ and energy $10^{18} \mathrm{GeV}$ are implications of much smaller size, faster time and higher energy that have not yet been identified with physical events.

Space size and time dependency. Nature possesses a time clock that is not unrelated to spatial size. Their interdependence can be observed from molecules that would coral for about $1 \mathrm{~s}$ within a circle of $1 \mathrm{~cm}$ in diameter. The ordinary notion of scaling specimen size and microstructure length being time independent is merely an approximation that ceases to hold at the atomic level. Atoms do collide and dissipate energy. These are firstorder effects at the nanoscale level.

On physical grounds, spatial size, rate effect, temperature and dissipation are combined to characterize the behavior of physical processes; variance of one would affect the others. Their combined effects have been quantified for problems in solids, liquids and gases by application of the nonequilibrium thermomechanics theory $[14,15]$ based on the concept of an isoenergy density space. This space is made of elements that do not shrink to zero. Their size and orientation change for each time-step and are determined from the displacement field by preserving the true nature of nonequilibrium. Finiteness of elemental size provides an account for the trade-off of surface and volume energy density, a fundamental aspect of spatial size effect that is not considered in classical continuum mechanics. The length dimension arising from strain gradients should be distinguished from that for the rate change of volume with surface. More discussion on this will be made in connection with generalizing the thermal conductivity expression [15] obtained by Boltzmann using statistical mechanics.

Scaling of energy dissipation. Bodies with large volume to surface ratio dissipate more energy by dilatation in contrast to distortion [16,17]. The dominance of dilatation tends to exhibit brittle behavior. As the volume to surface ratio is decreased, energy dissipation via distortion would overtake that of dilatation and a unit volume of 
the same material becomes more ductile. The ductile-brittle transition depends on the material microstructure, specimen shape and size, temperature and rate of deformation; it serves as a basis of reference for the design of structural members. Regardless of nonlinearity, the proportion of energy expanded by dilatation and distortion can be assessed from the stationary values of the energy density function $[18,19]$. Dominance by dilatation or distortion is a concept that remains to be validated for analyzing nanosize materials.

To elucidate the initial source of driving force for a line crack, a molecular dynamics region $[20,21]$ was inserted into the crack tip region surrounded by a field that uses continuum mechanics. The interface boundary is matched by invoking continuity of stresses and displacements. In addition, a system of forked slip lines is inserted as a source of dislocation emission driven by shear action. Recent findings from piezoelectric continuum mechanics and energy density theory $[18,19]$ have predicted (not preassumed) that a bifurcation damage pattern could prevail very close to the crack tip. The commonly known macrocrack growth accompanied by plastic enclaves to the side can still be predicted at distance away from the crack tip region. Both damage patterns are determinable from the same continuum mechanics formulation that is not restricted to any size scale if the prevailing physical constants are left undefined. This is not surprising because the material structure properties from piezoelasticity differ with distance from the crack tip. This makes the proportion of dilatational and distortional energy density distance dependent. The multiscale feature is exhibited in Fig. 1(a) at the microscopic scale and Fig. 1(b) at the macroscopic scale. The bifurcation damage pattern in Fig. 1(a), however,
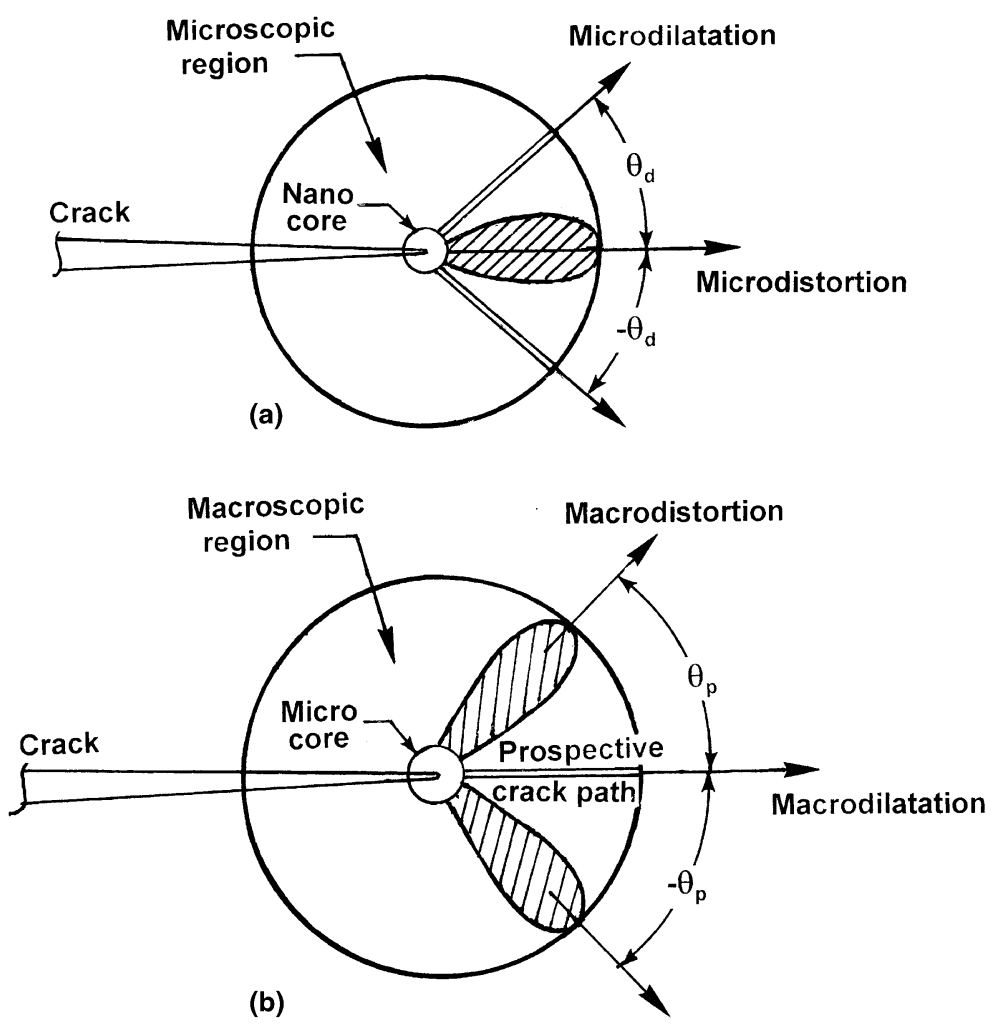

Fig. 1. Multiscale damage patterns at microscopic and macroscopic scales ahead a crack in piezoelastic material: (a) microscopic scale; (b) macroscopic scale. 
has a different interpretation than the dislocation slip lines invoked in [20,21]. They correspond to locations $\pm \theta_{\mathrm{d}}$, where dilatation would dominate. For the lack of a better terminology, they are referred to as lines of microdilatation. A single enclave of microdistortion lies along the line of symmetry. The familiar macrodamage pattern is displayed in Fig. 1(b). Macrodistortion zones appear at $\pm \theta_{\mathrm{p}}$ as plastic enclaves while the single macrodilational path coincides with the prospective site of macrocrack growth. Refer to [22,23] for details.

Necessary to hypothesize. A true description of nature, whether "corpuscular" and/or "continuous" does not appear to be in sight despite the advances made by scientists in modern and past times. Still remain in doubt are the size, shape, motion and property of the microparticles and the nonperceptible form of an energized cosmos. The fact may stand that it is not necessary to know how nature really behaves; it suffices to account for how nature might behave. This accounting procedure, however, must be executed with consistency and free of contradictions. It may rely on postulating a hypothesis that is not couched in the teleological language of final causality. Moreover, not all hypotheses should be granted with equal status, particularly to those invented just to explain one particular phenomenon. A useful hypothesis should account for a wide variety of facts successfully. Only then, it is not likely that faulted hypotheses would deduce all the observed facts. A single contradiction suffices to disqualify a hypothesis that may have satisfied other constraints.

The hypothesis that a material should break in a direction normal to the maximum stress seems to be self-explanatory. It encounters contradiction when a crack moves. The near tip maximum normal stress will then be parallel rather than normal to the prospective crack path. The preference, therefore, would be a hypothesis that could predict the correct crack path for both stationary and moving crack [24-26]. The same applies to predicting the location of crack initiation that occurs at the center of a specimen under static load and next to the surface under cyclically repeated load [27]. The energy density criterion [28,29] has the ability to account for failure by crack initiation and propagation for a wide cross-section of the available observed data at hand [30].

The foregoing discussions even though divided into topics, the underlying physics are related at the nanoscale. The different atomistic models [5-9] used to simulate physical processes have some profoundly disquieting features, not least of which is how the field and particle approaches are joined arbitrarily [20,21]. They are both assumed to be valid and used interchangeably. Discomforting as it may be in concept, this duality will be accepted as a prioristic practice.

\section{Remarks on particle and field concept}

Whether matter is continuous or corpuscular has been debated since the time of Aristotle (4th century). For every argument against one of the concepts, an equally convincing argument can be found to support it. Traditional natural philosophy in China (2nd century or earlier) envisaged the universe as a continuous medium or matrix in which all things possessed intrinsic rhythms. They interact through pulsations by radiating mutual influences and belong to parts in a hierarchy of organisms forming a cosmic pattern. The universe was thought to be engulfed by Chhi activated by wave-like progression of Yin and Yan forces that ascend from earth and descend from heaven; they come in contact and trigger the creation of all things.

Strange similarities in support of a continuum universe can be found in the philosophy of the Stoics in Mediterranean antiquity. These early views of Chinese and Greeks, however, were not quantified mathematically and applied systematically to explain physical phenomena until wave theories of light, heat and sound began to be developed in Europe.

\subsection{Individual field theories}

Modern particle physics, on the other hand, aims to unravel the basic building blocks of matter by attempting to observe shorter and shorter distances with higher and higher energy beams. Unsatisfying is the fact that the development resulted 
in four separate force field theories: the strong nuclear, weak nuclear, electromagnetic and gravitational. Unification became a central issue for three of the force fields leaving out the gravitational. Convergence is thought to occur when their energy intensities become equal. This presumably would take place around $10^{15} \mathrm{GeV}$ that is three orders away from the Planck energy $10^{18} \mathrm{GeV}$. Over the past 50 years, attempts have been made to build beam-type accelerators with increasing energy. Table 1 summarizes the few of the major accelerators that clearly attempt to approach the unification energy level stopping short at the energy level $\mathrm{TeV}$ anticipated by the superconducting supercollider (SSC) in Texas. The project has since been terminated. Even if the SCC were built, it would still be $10^{9} \mathrm{GeV}$ short of unification while the gravitational force field is still not included.

There are also conceptual difficulties associated with space-time point in quantum gravity theory where events are visualized as the exchange of virtual particles supported by interacting forces. These particles were assumed to be generated spontaneously out of the void and instantly vanish into the void. This sudden appearance and disappearance of particles remain as conjectural.

\subsection{Continuum space}

Matter can also be thought to constitute by regions of space in which the field is extremely intense. This idea is implicit in Chinese philosophy of the Tao which regards physical things and phenomena as transient manifestations. It embarks on the notion of emptiness and formless of Chhi which literally means "gas" or "ether", a term used in ancient China to denote the vital breath or energy animating the cosmos. It conceived as a tenuous and nonperceptible form of matter which is present throughout space and can condense into material objects. The latter tends to implicate the particle concept of quantum mechanics although no direct association were made with the discretization of matter into particles.

Ascendency and descendency of Yin and Yan forces assessed in strength are assumed to undergo four phases of changes in nature; they led to the notion of "harmonic motion" in physics. The concept was needed for inventing the clock. The four phases alter in succession as segments on a sine curve illustrated in Fig. 2. Table 2 outlines the cyclic behavior of nature in the "Book of Changes" [31].

The cyclic representation of natural phenomena has been identified with the observed reincarnation behavior of a white dwarf. Transformation of a white dwarf (supernova 1) can be described by the second columns in Table 2: shelter, destruction, chaos (or emptiness) and redifferentiation. These events described in Table 3 are illustrated in Figs. 3(a)-(d) inclusive. The conception of wave mechanics and field theories could not have originated without the notion of repeated changes with varying strength in the Book of Changes.

Table 1

Major accelerators built over the past 50 years

\begin{tabular}{llll}
\hline Accelerator & Approx. dates & Beam type & Approx. highest energy \\
\hline Brookhaven cosmotron & $1952-1967$ & Proton & $3 \mathrm{GeV}$ \\
Berkeley bevatron & $1954-1972$ & Proton & $6.4 \mathrm{GeV}$ \\
Cern proton synchrotron & 1960 & Proton & $30 \mathrm{GeV}$ \\
Brookhaven alternatory gradient synchrotron & 1960 & Proton & $33 \mathrm{GeV}$ \\
Slac 2 mile linear accelerator & 1966 & Electron & $30 \mathrm{GeV}$ \\
Fermilab accelerator & 1972 & Proton & $400 \mathrm{GeV}$ \\
Slac positron-electron asymmetric ring (spear) & 1972 & Electron-positron & $4.2-4.2 \mathrm{GeV}$ \\
Cornell electron storage ring (CESR) & 1978 & Electron-positron & $8-8 \mathrm{GeV}$ \\
DESY (Hamburg) tandem ring accelerator & 1979 & Electron-positron & $19-19 \mathrm{GeV}$ \\
Desy (Hamburg) tandem ring accelerator & 1979 & Electron-positron & $19-19 \mathrm{GeV}$ \\
Cern proton-antiproton collider & 1981 & Positron-antiproton & $270-270 \mathrm{GeV}$ \\
Superconducting supercollider (uncompleted) & 1986 & & $\mathrm{TeV}$ \\
\hline
\end{tabular}




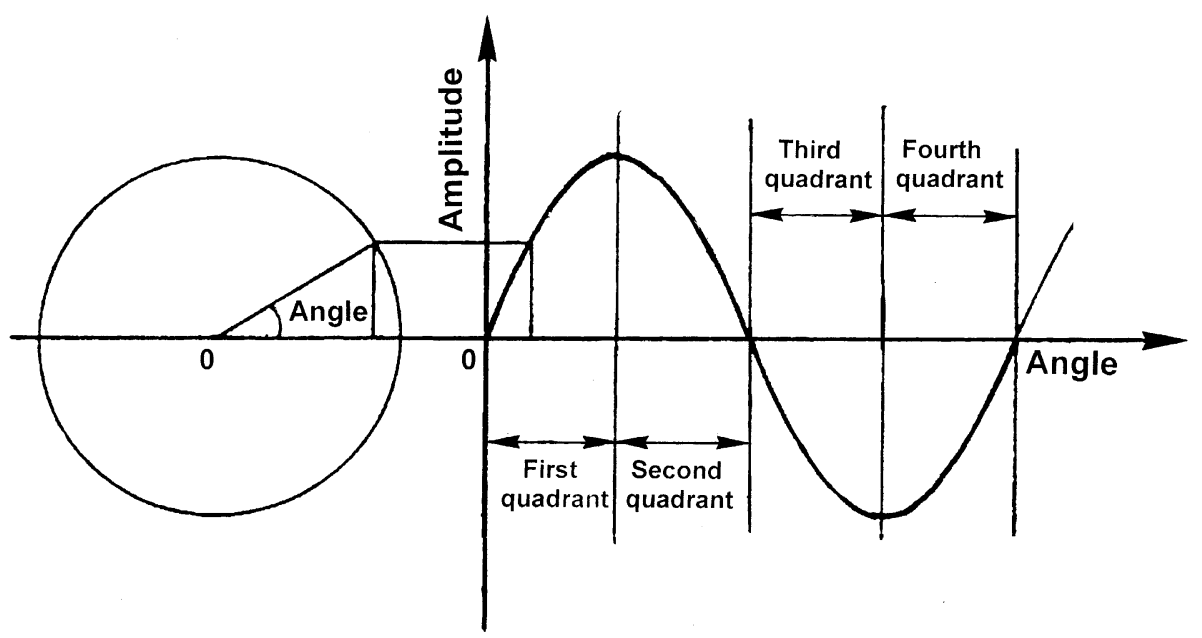

Fig. 2. Harmonic motion identified with phases of changes.

Table 2

Cyclic behavior driven by Yin and Yan forces

\begin{tabular}{lllll}
\hline Driving force & Four phases of change & & \\
\hline First quadrant (positive increase) & Spring & Shelter & Inception & Stability \\
Second quadrant (positive decrease) & Summer & Destruction & Climax & Impasse \\
Third quadrant (negative increase) & Autumn & Chaos (or emptiness) & Balance & Change \\
Fourth quadrant (negative decrease) & Winter & Redifferentiation & Anticlimax & Penetration \\
\hline
\end{tabular}

\subsection{Particle-continuum approach}

It is not inconceivable that the particle and field are not mutually exclusive; they could be thought as two aspects of the same reality that is in continual operation. At least, it can serve as a conceptual tool, no less incomprehensible than a limit approaching zero.

Movement of a macroscopic body is too austere to yield detail features of microparticles. However, the opposite is not true. The implication of Brownian motion has motivated the development of the nonequilibrium thermomechanics theory $[14,15]$ to relate temperature directly to the displacement or its gradient

$\frac{\mathrm{d} \Theta}{\Theta}=-\lambda \frac{\mathrm{d} V}{\mathrm{~d} A} \frac{\mathrm{d} e}{\mathrm{~d} \mathscr{D} / \mathrm{d} e}$,

in which $\Theta$ stands for the absolute temperature and $e$ the displacement gradient. The negative sign refers to work done on the system. The change of volume with that of surface $\mathrm{d} V / \mathrm{d} A$ can be ex- pressed explicitly in terms of displacement gradients. Eq. (1) is referred to the isoenergy density space. The dissipation energy density function $\mathscr{D}$ can be measured experimentally $[32,33]$ or computed from a numerical algorithm developed for mechanical unloading [34]. The temperature $\Theta$ can then be obtained from Eq. (1) without using theories of classical heat transfer and thermodynamics. Besides, it applies to all the force fields: Strong nuclear, weak nuclear, electromagnetic and gravitational with $\lambda$ being a weighting factor as defined in Table 4.

A departure from the traditional approach is that $\lambda(\mathrm{d} V / \mathrm{d} A)$ in Eq. (1) may be regarded as a special form of the more general function $f(e)$ that depends only on the isostrain $e$ or displacement gradients referred to the isoenergy density space. With this understanding, the volume energy density can be written as

$\frac{\mathrm{d} W}{\mathrm{~d} V}=\iint f(e) \mathrm{d} e \mathrm{~d} e$. 
Table 3

Reincarnation of a white dwarf

\begin{tabular}{ll}
\hline Phase change & Description of transformation \\
\hline Shelter: Fig. 3(a) & $\begin{array}{l}\text { A white dwarf is attracted into the orbit of an ordinary star } \\
\text { A process of destruction occurs when the hydrogen and } \\
\text { helium envelopes of white dwarf (now a red giant) expand and } \\
\text { overflow onto the less massive star until the process of } \\
\text { overflow is reversed } \\
\text { As the mutual destruction process repeats, the temperature in } \\
\text { the white dwarf increases to } 10^{3} \text { million degrees creating the } \\
\text { "carbon detonation" and disintegrates }\end{array}$ \\
$\begin{array}{ll}\text { A runaway star is thus released like a sling shot and the } \\
\text { process is repeated. }\end{array}$ \\
\hline
\end{tabular}

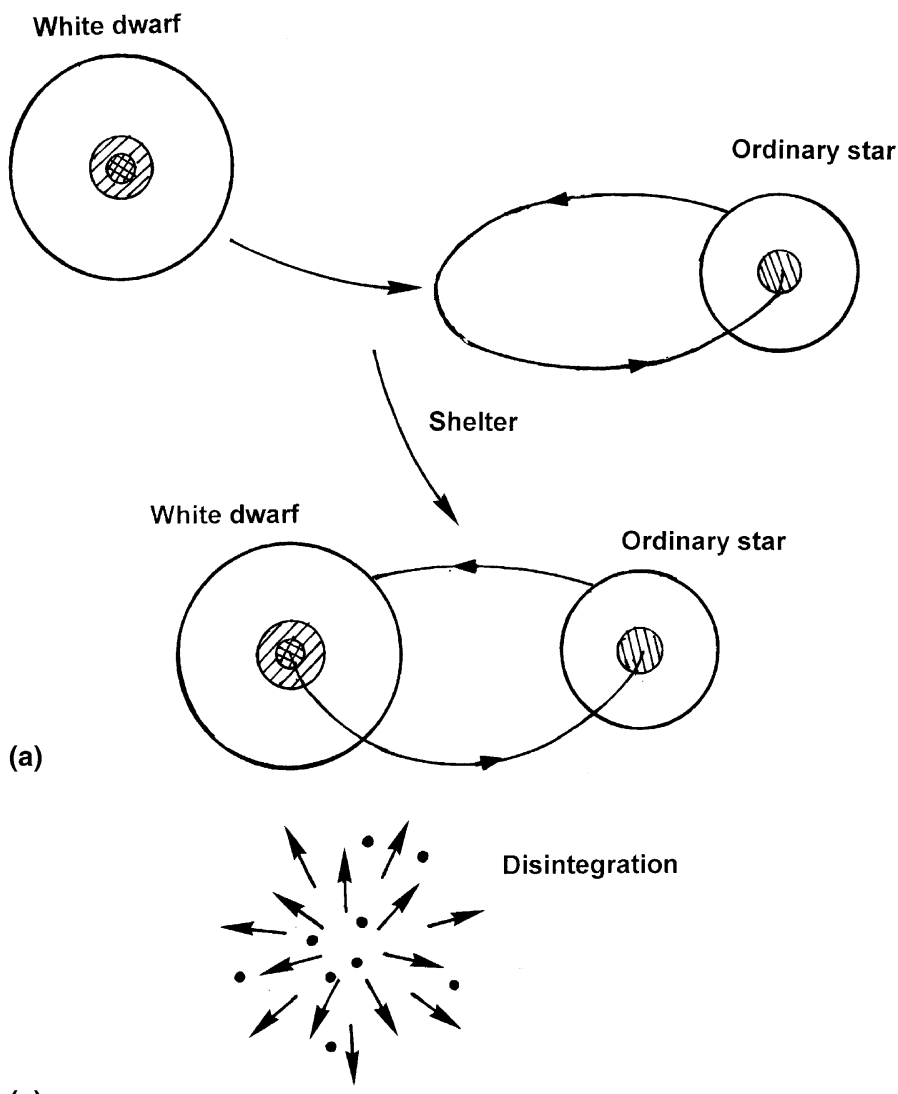

(c)

White dwarf

(b)

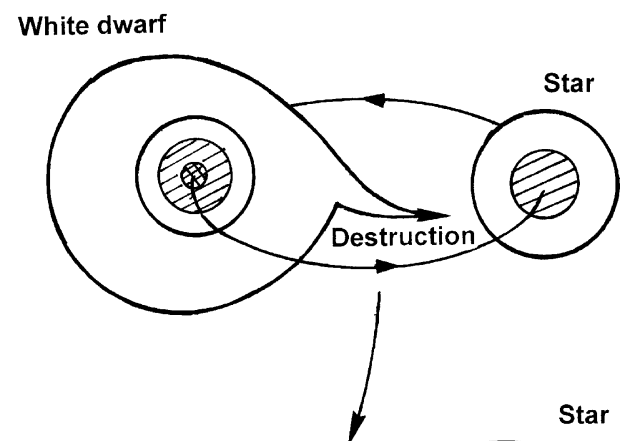

(d)

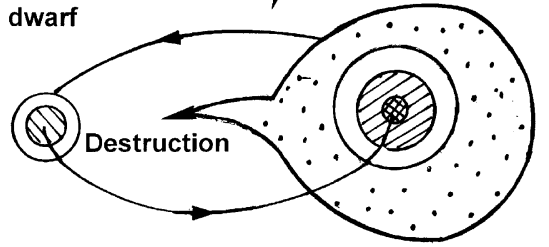

Fig. 3. Transformation phase of a white dwarf: (a) first phase: shelter; (b) second phase: destruction; (c) third phase: chaos and emptiness; (d) fourth phase: redifferentiation.

The more general representation of $f(e)$ or $\mathrm{d} V / \mathrm{d} A$ against the isostrain $e$ in Fig. 4 includes the specific cases of stress $\sigma$ versus strain $\epsilon$ in elasticity, electric displacement $D$ versus electric field $E$ in electroelasticity, flux density $B$ versus magnetic field $H$ in magnetoelasticity, etc. The symbol $\epsilon$ applies to uniaxial strain while the isoenergy strain $e$ can be transferred to the physical space to yield 
Table 4

Weighting factor for force fields

\begin{tabular}{ll}
\hline Force field & Application and spatial range \\
\hline Strong nuclear- $\lambda_{\mathrm{I}}$ & $\begin{array}{l}\text { Nuclear bond, fission and } \\
\text { fusion: } 10^{-13} \mathrm{~cm}\end{array}$ \\
Electromagnetic- $\lambda_{\mathrm{II}}$ & $\begin{array}{l}\text { Electricity, magnetism and } \\
\text { light: infinite }\end{array}$ \\
Weak nuclear- $\lambda_{\mathrm{III}}$ & Radioactive decay: $10^{-16} \mathrm{~cm}$ \\
Gravitational- $\lambda_{\mathrm{IV}}$ & $\begin{array}{l}\text { Planetary orbits and motion of } \\
\text { stars: infinite }\end{array}$ \\
\hline
\end{tabular}

displacement gradients in three dimensions. The nearly flat portion of the curve in Fig. 4 corresponds to linearity between $\sigma$ and $\epsilon, D$ and $E$ or $B$ and $H$. There would be no loss in generality as all physical events could be quantified as wave-like disturbances in matter regardless of the type of force field indicated in Table 4. Motion of the constituents are assumed to be activated by forces, however, defined as long as their characteristic length and wave speed are correctly identified. Phenomena that are detectable by light would be associated with characteristic length of $10^{-5}$ $10^{-6} \mathrm{~cm}$ and wave speed of $3 \times 10^{8} \mathrm{~m} / \mathrm{s}$ while those by sound would involve characteristic length of $10^{-3} \mathrm{~cm}$ and wave speed of $10^{2}-10^{3} \mathrm{~m} / \mathrm{s}$. No ambiguity would arise in the assessment of physical events if the mutual interaction of size and time are included. Temperature change is also needed to account for energy dissipation.

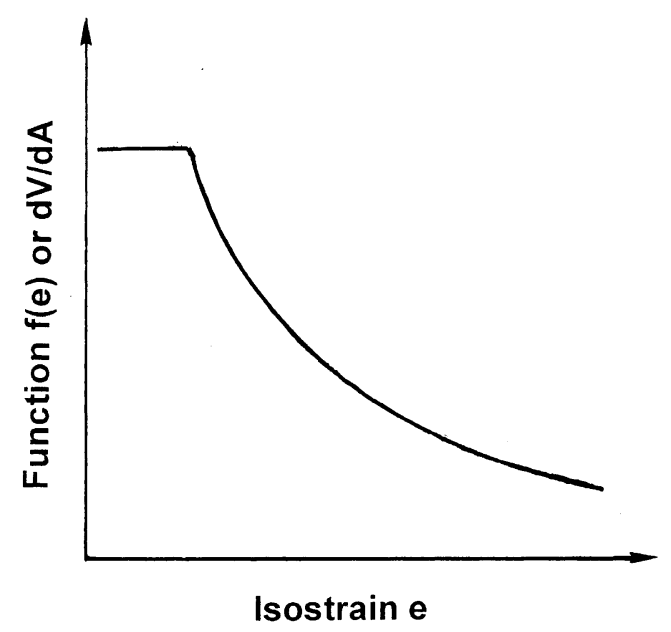

Fig. 4. Variations of strain function with isostrain.
To reiterate, displacements are the primary quantities in the isoenergy density formulation [15]. Once known, the temperature follows from Eq. (1) based on the premise that the dissipation energy density $\mathscr{D}$ can be found. This requires a physical interpretation of system irreversibility. To begin with, the dissipation energy density is identified with the unrecoverable portion of $\mathrm{d} W / \mathrm{d} V$ caused by permanent change in the material microstructure and possibly the atomic or subatomic structure. The interest lies in quantifying $\mathscr{D}$ analytically and/or detecting it experimentally. It turns out that the different propositions for evaluating $\mathscr{D}$ do not affect the results appreciably as long as the trend of $\mathscr{D}$ varying with time is correctly recognized.

Analytical unloading. This scheme involves applying a mechanical load or other form to negate that applied at the previous time-step to simulate unloading. The difference is considered to be the dissipation. While the number of loading and unloading steps tend to make a difference on the outcome, consistency takes precedency. This procedure has been applied to solve a host of physical problems that are inherently nonequilibrium [34-42]. This could involve phase transformation owing to excessive local energy transfer.

Experimental strain-based measurement. An experimental procedure has been developed by the US Naval Research Laboratory (NRL) to measure the dissipated energy density [32,33] that corresponds precisely to $\mathscr{D}$ in Eq. (1). It was originally intended for characterizing the internal damage of cracked composite specimens under load. Extensive data have since been obtained using the NRL In-Plane Loader [33] which is a computer-controlled testing machine capable of producing multiple combinations of opening/closing, sliding and rotating boundary data which can be converted to compute the dissipated energy density. This involves matching a function of strain with other functions containing coefficients that are evaluated by minimizing the difference between the experimentally measured and analytically estimated energy density dissipation function. Details of the test apparatus and the associated numerical algorithms can be found in [33]. Design 
applications to naval structures have been made; they consist of a ship mast and submarine hull.

\section{Dependency of thermal energy transmission on size scale}

Mathematical and computer modeling have shown that nanomaterials and nanodevices could possess the advantage of superior strength and high speed. Such features would make them technically and economically desirable for a new range of applications. This potential has provided the incentives for engineers to have an understanding of quantum mechanical effects.

\subsection{Contribution of electrons and phonons to thermal conduction}

Elections and phonons are postulated to be the respective lattice vibration quanta that move from hot to cold over a large number of atomic distances to conduct heat. Electrons would be dominant in the heat transfer process for metallic materials while phonons for dielectric materials. Expressed in terms of heat capacity for constant volume, the electron contribution refers to

$C_{v}^{\mathrm{el}}=\frac{\pi^{2} N_{0} k_{\mathrm{b}}^{2} T}{2 E_{\mathrm{f}}}$

such that the heat capacity $C_{v}^{\mathrm{el}}$ depends linearly on the temperature $T$ in $\mathrm{K}$. The Fermi energy is $E_{\mathrm{f}}$. In Eq. (3), $N_{0}=6.022 \times 10^{23} \mathrm{~J} / \mathrm{K}$ is the Avogadro constant and $k_{\mathrm{b}}=1.381 \times 10^{-23} \mathrm{~J} / \mathrm{K}$ is the Boltzmann constant. Statistical mechanics gives a relation between the thermal conductivity $k^{\mathrm{el}}$ and heat capacity per unit volume $C_{v}^{\mathrm{el}}$ as

$k^{\mathrm{el}}=\frac{1}{3} C_{v}^{\mathrm{el}} v^{\mathrm{el}} \ell^{\mathrm{el}}$,

while $C_{v}^{\mathrm{el}}$ increases with $T$. Both the velocity $v^{\mathrm{el}}$ and mean free path $\ell^{\mathrm{el}}$ of the electrons decrease with $T$. Hence $k^{\text {el }}$ remains nearly unchanged. Only those electrons which have an energy close to the Fermi energy $E_{\mathrm{f}}$ are able to participate in the conduction process. Therefore, $v^{\mathrm{el}}$ is essentially the Fermi velocity $v_{\mathrm{f}}$ in the calculation
$E_{\mathrm{f}}=\frac{1}{2} m v_{\mathrm{f}}^{2}$.

The heat conductivity $k^{\text {el }}$ associated with the electrons in Eq. (4) is a scalar. A general expression of $k^{\mathrm{el}}$ can be derived for all three orthogonal directions and expressed in terms of a dissipation energy density capacity $C_{\Theta}$, the rate change of volume with surface $\mathrm{d} V / \mathrm{d} A$ and velocity components $\mathrm{d} x_{i} / \mathrm{d} t(i=x, y, z)$. A series of experiments would have to be performed [33] to obtain the function $\mathscr{D}$ as a function of the temperature from which $C_{\Theta}$ can be evaluated as $\mathrm{d} \mathscr{D} / \mathrm{d} \Theta$. The symbol $T$ for temperature shall be distinguished from $\Theta$ in the isoenergy density theory where the effect of $\mathrm{d} V / \mathrm{d} A$ is considered. More discussion on this will follow.

In the same vein, the quantum mechanics approach can also be applied to derive an expression for the lattice heat capacity per volume of the phonons:

$C_{v}^{\mathrm{ph}}=3 N_{0} k_{\mathrm{b}} T_{\mathrm{e}} \frac{\exp T_{\mathrm{e}}}{\left(\exp T_{\mathrm{e}}-1\right)^{2}}$,

in which $T_{\mathrm{e}}$ is defined as the Einstein temperature

$T_{\mathrm{e}}=\frac{h \omega}{2 \pi k_{\mathrm{b}} T}$.

The vibration frequency of phonons is $\omega$ and $h=6.626 \times 10^{-34} \mathrm{~J} \mathrm{~s}$ is the Planck constant. Eq. (6) reduces to an exponential form for very small temperature. This does not agree with the experimental finding that $C_{v}^{\text {ph }}$ decreases by $T^{3}$. Debye has corrected this shortcoming but is not of direct concern for this discussion because Eq. (6) can be bypassed when the thermal conductivity for the flow of phonons

$k^{\mathrm{ph}}=\frac{1}{3} C_{v}^{\mathrm{ph}} v^{\mathrm{ph}} \ell^{\mathrm{ph}}$

will be replaced subsequently by a more general form of Eq. (8) that will not involve $C_{v}^{\mathrm{ph}}$ while the phonon velocity will have three components. On the average, the phonon velocity $v^{\mathrm{ph}}$ is about the same as the sound velocity $5 \times 10^{5} \mathrm{~cm} / \mathrm{s}$ and is relatively temperature independent. The phonon mean free path $\ell^{\text {ph }}$ is about $10 \mathrm{~nm}$ at room temperature to $10^{4} \mathrm{~nm}$ near $20 \mathrm{~K}$. Phonons produce atomic displacements giving them a transient like 
or nonequilibrium character. If the environment temperature is increased phonons tend to flow into the crystal. The increased atomic vibrations will in turn raise the crystal's temperature. Conversely, decreasing the environment temperature enhances phonon emission from the crystal. These properties of phonon flow could enhance or impede thermal conductivity.

\subsection{Phonon imperfection}

The excitation of electrons and phonons lead to atomic displacements. In this sense, they may be regarded as imperfections known as electronic imperfections and phonon imperfections. Presumably, they would affect the strength of crystals as dislocations have in connection with plasticity although the few scanty available research works in this area are concerned with the electrical properties of semi-conductors rather than with material science. Interaction of phonons with an imperfection has been examined in [43]. Molecular dynamics was used to obtain the atomic velocities, forces and potential energies for exposing the presence of localized phonon-like modes near the tip of a moving crack prior to dislocation emission and crack bifurcation. Bond breaking energy ahead of a fast moving crack tends to excite local phonons that travel as sound waves. The thermally activated process ahead of the crack is length scale dependent. Ledges and other defects on crack front border could also act as dislocation nucleation sources that are detectable by high resolution transmission electron microscopes. There are evidence of dislocation loops emitting from the front of an embedded crack. The implications of threedimensional effects in contrast to those in two dimensions may affect interpretation of the mechanical strength and/or electrical properties of crystals.

Detail features of phonon spectrum have also been related to the dynamical properties of adsorbate covered surface of solids [44,45]. They provide insight into the bonding properties between absorbates and substrates. Phonon dispersion curves have been obtained from high resolution electron energy loss spectroscopes. Theoretical calculations have also been made by using the embedded atom method to check the experimental surface phonon dispersion curves.

\subsection{Temperatures change with volume}

Kevin's law enunciated more than 140 years ago in 1857 [46] continues to dominate the thought that temperature change $\Delta T$ is related exclusively to volume change $\Delta V$. This influence prevailed not only in the thermal conductivity derivation of Boltzmann but also on Weibull's statistical treatment $[47,48]$ of specimen size effects. Only the specimen volume entered in to the correlation. More specifically, Kelvin's equation shows that

$\frac{\Delta T}{T}=-Y \frac{\Delta V}{V}$.

Here, $V$ is the volume and $Y$ the Gruneisen parameter given by $\alpha K_{\mathrm{T}} / c_{v}$. The quantity $K_{\mathrm{T}}$ is the isothermal modulus of compressibility and $c_{v}$ the specific heat per unit volume at constant volume. The cubical dilatation of volume $\Delta V / V$ is equal to $(1-2 v) \epsilon$ with $v$ being Poisson's ratio and $\epsilon$ the uniaxial strain the loading direction. In effect, Eq. (9) considers only dilatation via $\Delta V / V$ and neglects distortion, i.e., the change of surface $\Delta A$.

Eq. (9) was originated empirically from monitoring temperature change in mild steel bars when stretched and compressed [46]:

$\frac{\Delta T}{T}=-\frac{\alpha \sigma}{2 c_{\sigma} J}$.

The symbol $\sigma$ in Eq. (10) stands for the suddenly applied stress within the elastic range of strain. Note that $\alpha$ is the coefficient of linear thermal expansion, $c_{\sigma}$ the heat required to raise temperature by one degree in a unit weight of substance under constant stress and $J$ the mechanical equivalent of heat. Temperatures were recorded [49] by thermocouples for three tensile and three compression tests. The results are displayed in Figs. 5(a) and (b). Sudden changes in the temperature curves were detected in the temperature curves of Figs. 5(a) and (b). Sudden changes in the temperature curves were detected in the temperature curves of Figs. 5(a) and (b). It was implied at the time that they would correspond to the instant at which the material 


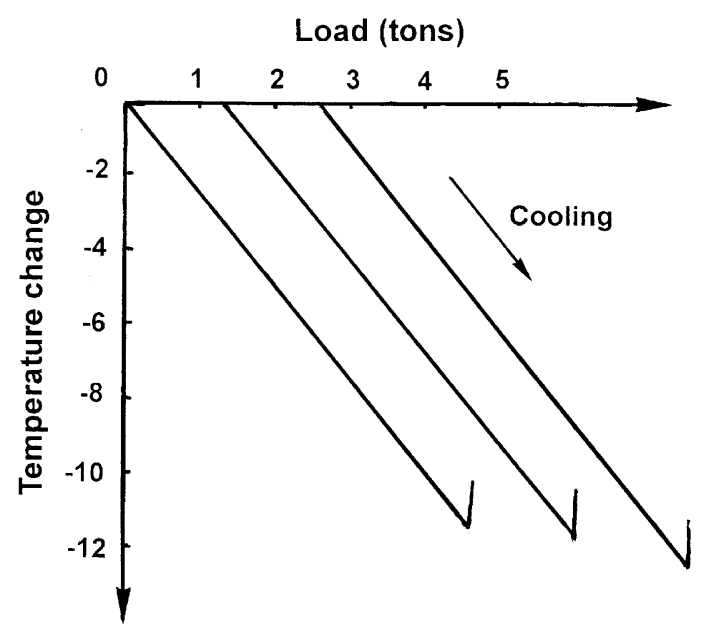

(a)

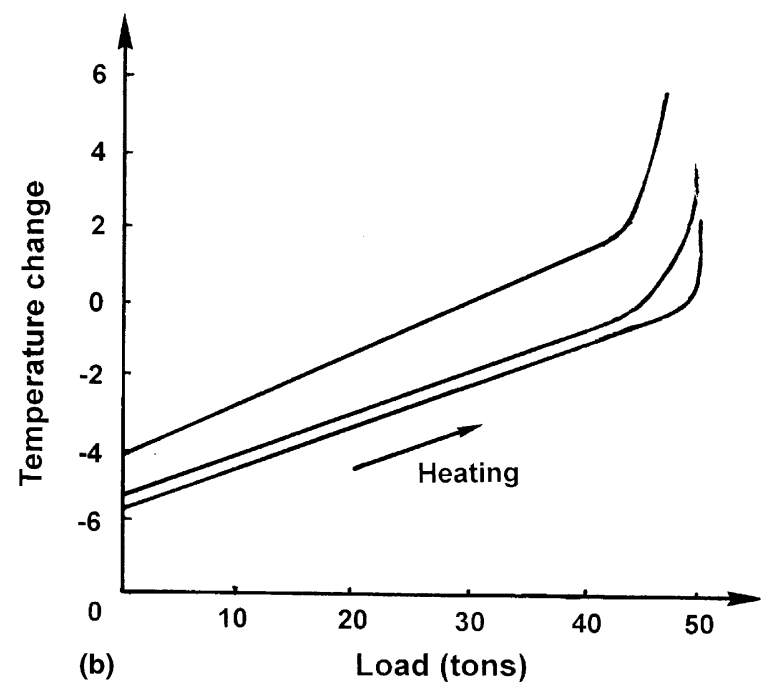

(b)

Fig. 5. Temperature change in mild steel bars on rapid loading [47]: (a) tension; (b) compression (tons).

yield point was reached. Such an explanation did not hold for the measurements made in [36,37] on steel and aluminum specimens with thermistors that have accuracies of $\pm 10^{-3} \mathrm{~K}$. Uniaxial tensile tests on SAFC-40R steel [36]; and 6061T6 aluminium [37] showed that irreversibility of the response is noticeable at the macroscopic scale in the linear portion of the stress and strain curve. It is conceptually incorrect to assume loading and unloading to occur on the same portion of a linear curve even if the difference is small. This effect, however, is not negligible on the time scale where the specimen temperature can remain below ambient for minutes depending on the applied displacement rate [37]. The temperature cooling and heating behavior are exhibited in Fig. 6(a) for 6061-T6 aluminum subjected to a displacement rate $8.467 \times$ $10^{-5} \mathrm{~m} / \mathrm{s}$. The corresponding stress and strain curves are displayed in Fig. 6(b). The point with
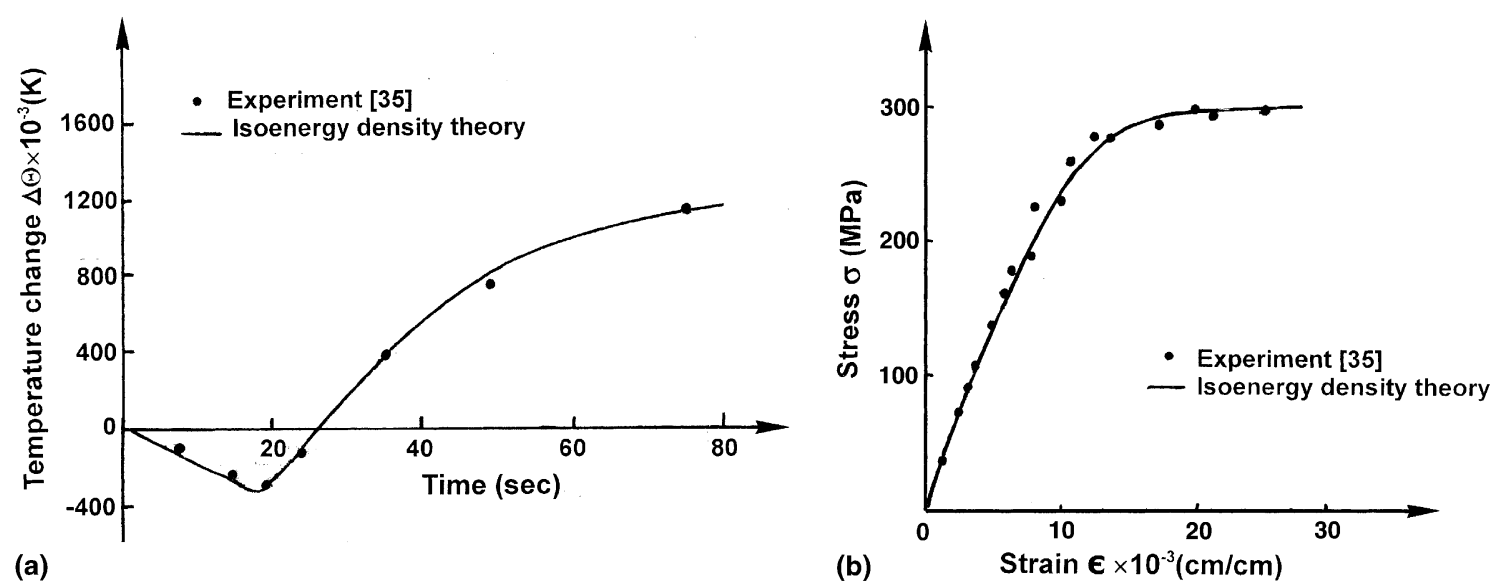

Fig. 6. Nonequilibrium temperature and stress-strain date for 6061 aluminum strip stretched at $8.467 \times 10^{-5} \mathrm{~m} / \mathrm{su}^{\mathrm{s}}(\mathrm{a}) \mathrm{cooling}$ and heating; (b) stress and strain curves. 
a stress of $292.0 \mathrm{MPa}$ and strain of $12.0 \times$ $10^{-3} \mathrm{~cm} / \mathrm{cm}$ at $t=45.12 \mathrm{~s}$ corresponds to the flat portion; it is no way near the location of $t \approx 20 \mathrm{~s}$ where the curvature of the temperature reverses. The argument that the yield point is associated with the trough in the temperature curve for mild steel does not seem to hold for aluminum. It should also be emphasized that the ascending portion of the curves in Fig. 6(a) from $t \approx 20$ to $80 \mathrm{~s}$ cannot be predicted from Kelvin's equation as a result of neglecting the effect due to surface change. If the applied displacement rate is slowed by one order of magnitude, it would require more than $200 \mathrm{~s}$ or about $3.3 \mathrm{~min}$ for the aluminum specimen to return to the ambient temperature after loading. This time lag is not insignificant and exhibits the nonequilibrium character of the uniaxial stress and strain data where thermal energy exchange between the system and its surrounding is the rule rather than the exception.

In retrospect, the classical theory of elasticity had to invoke a closed thermodynamic system that is isolated from thermal fluctuation of the environment. This circumvents the formulation of a nonequilibrium theory of continuum mechanics. Consistency of classical continuum mechanics formulation is thus achieved at the expense of decoupling the thermal and mechanical effects by letting the rate change of volume with surface to approach zero or $\mathrm{d} V / \mathrm{d} A \rightarrow 0$. The divorce of thermal change from mechanical deformation necessitates the development of heat transfer theory for finding temperature change. Vanishing of $\mathrm{d} V / \mathrm{d} A$ implies that the thermal conductivity in Fourier's law of heat conduction, the Stefan-Boltzmann constant in heat radiation and the convective heat transfer film coefficient shall all in the limit go to zero. The finiteness of $\mathrm{d} V / \mathrm{d} A$ must be preserved because surface is an integral part of the body. Surface energy and volume energy cannot be unconnected. Their exchange determines the size of a crystal in the nucleation process that is well known. Size scaling in continuum mechanics is also being denied because of the limit $\mathrm{d} V / \mathrm{d} A \rightarrow 0$. This implies that the continuum element has no size and therefore no shape.

\subsection{Coexistence of volume and surface}

Surface and volume being an entity are descriptive of configurations in three dimensions as length is in one dimension. They expand or contract in harmony. Such configural changes occur for each element of the matter when disturbed. This process gives rise to thermal fluctuations such that no single deformation and/or temperature is representative of the matter as a whole. This is the true character of nonequilibrium in nature.

On physical grounds, the rate changes of volume with surface $\mathrm{d} V / \mathrm{d} A$ cannot vanish. In three dimensions with $i=x, y, z$, there prevails one component of $\mathrm{d} V / \mathrm{d} A$ in each of the three directions. It can thus be postulated that

$$
\left(\frac{\mathrm{d} W}{\mathrm{~d} A}\right)_{i}=\left(\frac{\mathrm{d} V}{\mathrm{~d} A}\right)_{i} \frac{\mathrm{d} W}{\mathrm{~d} V}, \quad i=x, y, z,
$$

in which $(\mathrm{d} W / \mathrm{d} A)_{i}$ are the components of the surface energy density $\mathrm{d} W / \mathrm{d} A$, a vector quantity while the volume energy density $\mathrm{d} W / \mathrm{d} V$ is a scalar.

Eq. (11) is associated with crystal nucleation. The change in free energy $\mathrm{d} F$ for the homogeneous nucleation of an embryo to the new phase can be expressed as the sum of the change in volume free energy $\mathrm{d} F_{V}$ and surface free energy $\mathrm{d} F_{A}$ required to form the new surface. For a spherical nucleus of radius $R$

$\mathrm{d} F=4 \pi R^{2} \mathrm{~d} F_{A}-\frac{4}{3} \pi R^{3} \mathrm{~d} F_{V}$.

A negative sign is necessary because the transformation proceeds from an unstable to stable state. The condition for continued growth of an embryo is that the radius should exceed $R_{0}$ such that first derivative of $\mathrm{d} F$ with reference to $\mathrm{d} R$ should vanish. This yields

$$
\left(\frac{R}{2}\right)_{R \rightarrow R_{0}}=\frac{\mathrm{d} F_{A}}{\mathrm{~d} F_{V}} .
$$

The critical free energy or activation energy for nucleation is given by

$\mathrm{d} F_{\mathrm{c}}=\frac{16 \pi}{3} \frac{\mathrm{d} F_{A}^{3}}{\mathrm{~d} F_{V}^{2}}$. 
Eq. (13) is in fact equivalent to Eq. (11) written in the $R$-direction in one dimension [50]:

$$
\left(\frac{\mathrm{d} V}{\mathrm{~d} A}\right)_{R}=\frac{(\mathrm{d} W / \mathrm{d} A)_{R}}{\mathrm{~d} W / \mathrm{d} V} .
$$

For a sphere, $V=3 / 4 \pi R^{2}$ and $V=4 \pi R^{2}$ lead to $\mathrm{d} V / \mathrm{d} A=R / 2$. In Eq. (15), $(\mathrm{d} W / \mathrm{d} A)_{R}$ and $\mathrm{d} W / \mathrm{d} V$ correspond, respectively, to $\mathrm{d} F_{A}$ and $\mathrm{d} F_{V}$. The point is that $\mathrm{d} V / \mathrm{d} A=R / 2$ cannot be zero because the exchange of surface and volume energy determines the crystal size. The crystal nucleation process would not be possible if $\mathrm{d} V / \mathrm{d} A$ is made to vanish.

Coexistence of volume and surface energy in the isoenergy density theory [15] has led to a deeper understanding of the heat transfer mechanisms in conduction, radiation and convection.

Heat conduction. The thermal conductivity $k_{i}$ in Fourier's law of heat conduction can be generalized to the form

$k_{i}=C_{\Theta}\left(\frac{\mathrm{d} V}{\mathrm{~d} A}\right)_{i}\left(\frac{\mathrm{d} x_{i}}{\mathrm{~d} t}\right), \quad i=x, y, z$,

in which $C_{\Theta}$ is the energy dissipation capacity coefficient defined as

$C_{\Theta}=\frac{\mathrm{d} \mathscr{D}}{\mathrm{d} \Theta}$.

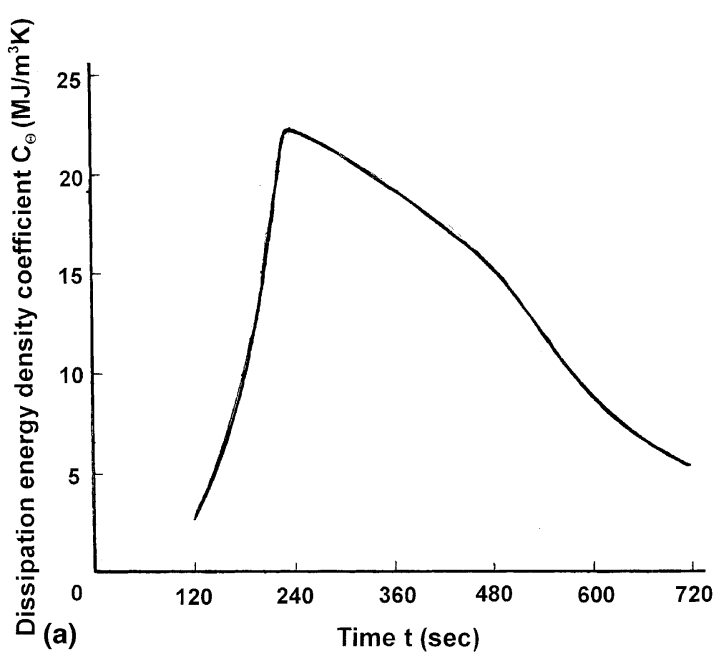

As mentioned earlier, $\mathscr{D}$ as a function of $\Theta$ can be evaluated experimentally from the method developed by NRL [32,33]. The problem of a 6061 aluminum cylindrical bar with a diameter of 1.25 and $10 \mathrm{~cm}$ in length is solved in an open environment consisting of air at room temperature $\left(25^{\circ} \mathrm{C}\right)$. The air medium $10 \mathrm{~cm} \times 10 \mathrm{~cm}$ is permitted to interact with the aluminum bar displaced at a rate of $1.27 \times 10^{-4} \mathrm{~cm} / \mathrm{s}$. Thermal changes across the solid/air interphase are evaluated by application of the isoenergy density theory. The objective here is to show that $C_{\Theta}$ in Eq. (17) together with $(\mathrm{d} V / \mathrm{d} A)_{i}$ and $\mathrm{d} x_{i} / \mathrm{d} t$ in Eq. (16) for $i=r, z$ can be computed analytically. Here, $r$ and $z$ denote, respectively, the radial and axial direction of the cylinder. Hence, the thermal conductivity $k_{r}$ (or $k_{z}$ ) is determinable from analysis. Such a capability is valuable as it could take place of experiments that are time consuming and expensive.

Fig. 7(a) shows that global average of the energy dissipation capacity coefficient as a function of time. It rises to a peak between 180 and $240 \mathrm{~s}$ and then decreases. Transient character of the global thermal conductivity $k_{r}$ (or $k_{z}$ ) is displayed in Fig. 7(b). The peak for $k_{r}$ occurs near $t=240 \mathrm{~s}$. As time increases, $k_{r}$ tends to equilibrium and is within the upper and lower bound values of 237 and $65 \mathrm{~W} / \mathrm{m} \mathrm{K}$ as reputed in the open handbooks.

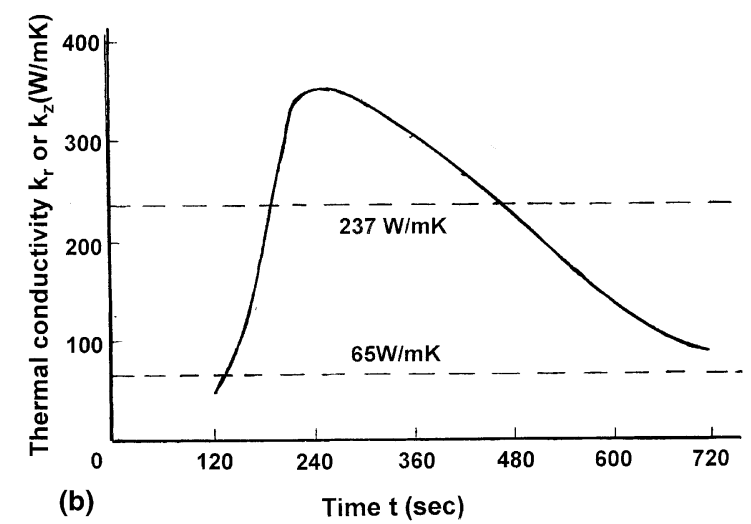

Fig. 7. Time history of global dissipation energy density coefficient and thermal conductivity at $25^{\circ} \mathrm{C}$ for 6061 aluminum bar stretched at $1.27 \times 10^{-4} \mathrm{~cm} / \mathrm{s}$ : (a) dissipation energy density coefficient; (b) thermal conductivity. 
Convective heat transfer. The film coefficient $h_{i}$ in convective heat transfer can also vary in the three orthogonal directions:

$h_{i}=C_{\Theta}\left(\frac{\mathrm{d} V}{\mathrm{~d} A}\right)_{i} \frac{1}{\mathrm{~d} t}, \quad i=x, y, z$.

Eq. (18) implies that convective heat transfer is connected with the exchange of surface and volume energy via $(\mathrm{d} V / \mathrm{d} A)_{i}$.

Since $C_{\Theta}$ is already known from Fig. 7(a) of the previous example the data can also be used to obtain $h_{r}$ averaged in the axial direction. The result is summarized in Fig. 8 for $h_{r}$ varying near the solid/air interphase. A mean value of $h_{r}$ equal to $205.9 \mathrm{~W} / \mathrm{m}^{2} \mathrm{~K}$ is obtained for a near wall film thickness of $0.785 \mathrm{~cm}$ which corresponds to the distance at which the area above and below the line $205.9 \mathrm{~W} / \mathrm{m}^{2} \mathrm{~K}$ would be equally divided.

Radiation heat transfer. The Stefan-Boltzmann coefficient $\eta_{i}$ in radiation heat transfer takes the form

$\eta_{i}=\frac{\mathrm{d} D}{\mathrm{~d} t}\left(\frac{\mathrm{d} V}{\mathrm{~d} A}\right)_{i} \frac{1}{\Theta^{4}-\Theta_{0}^{4}}, \quad i=x, y, z$

The validity of Eq. (19) was made by determining the radiation energy emitted by microstructure

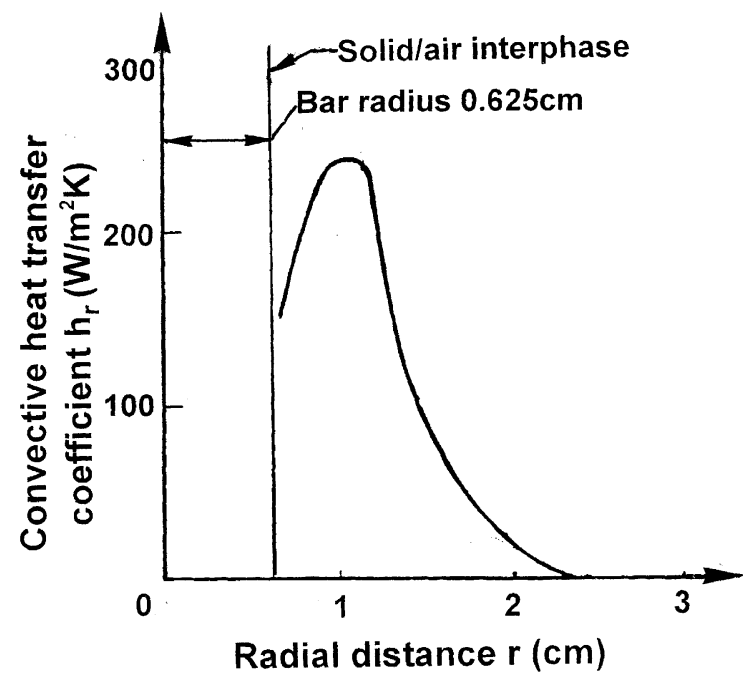

Fig. 8. Radial variations of convective heat transfer coefficient averaged in axial direction at $25{ }^{\circ} \mathrm{C}$ for 6061 aluminum bar stretched at $1.27 \times 10^{-4} \mathrm{~cm} / \mathrm{s}$. phase transformation during high speed impact of a tungsten projectile on a 4340 steel target plate [51]. The projectile is $14 \mathrm{~cm}$ in width and $47 \mathrm{~cm}$ in length and the target is $20 \mathrm{~mm}$ thick and $60 \mathrm{~mm}$ wide. It is advanced in small time increments into the plate until complete penetration of the target is achieved. This occurred at $\Theta=892.73 \mathrm{~K}$ corresponding approximately to $30.31 \mu \mathrm{s}$. A plug of target material is formed and leaves the plate. The narrow shear bands found in region next to the plug has been named as "adiabatic shear bands" based on the speculation that there is no time for heat transfer by conduction to take place. The austenization of the 4340 steel in a very narrow band of material during plug formation, however, has been identified with heat dissipation by radiation which can occur at a speed much faster than conduction. Therefore, adiabatic is not an appropriate description of the process. This is confirmed from the Stefan-Boltzmann coefficients in the axial and transverse direction of the projectile; they are computed to be $\eta_{z}=5.1312 \times 10^{-8} \mathrm{~W} / \mathrm{m} \mathrm{K}$ and $\eta_{r}=5.08 \times 10^{-8} \mathrm{~W} / \mathrm{m} \mathrm{K}$. This agrees well with the average handbook value of $\eta=$ $5.669 \times 10^{-8} \mathrm{~W} / \mathrm{m} \mathrm{K}$. The heat conductivity coefficients $k_{r}$ and $k_{z}$ computed from the data for plug formation were not close to the average handbook value. This is an indication that heat transfer by conduction is not the process.

\subsection{Generalized thermal conductivity for electrons and phonons}

In view of Eq. (16), the expression $k^{\mathrm{el}}$ in Eq. (4) and $k^{\mathrm{ph}}$ in Eq. (8) can be generalized to the forms:

$k_{i}^{\mathrm{el}}=C_{\Theta}^{\mathrm{el}}\left(\frac{\mathrm{d} V}{\mathrm{~d} A}\right)_{i}^{\mathrm{el}}\left(\frac{\mathrm{d} x_{i}}{\mathrm{~d} t}\right)^{\mathrm{el}}, \quad i=x, y, z$

and

$k_{i}^{\mathrm{ph}}=C_{\Theta}^{\mathrm{ph}}\left(\frac{\mathrm{d} V}{\mathrm{~d} A}\right)_{i}^{\mathrm{ph}}\left(\frac{\mathrm{d} x_{i}}{\mathrm{~d} t}\right)^{\mathrm{ph}}, \quad i=x, y, z$.

Eqs. (20) and (21) imply that electrons and phonons can travel in three directions with different velocities. Since their displacements could also differ in the $x, y$ and $z$ directions, the change of volume with surface $\mathrm{d} V / \mathrm{d} A$ would alter 
accordingly. It plays the role of the mean free path $\ell^{\mathrm{el}}$ in Eq. (4) and $\ell^{\mathrm{ph}}$ in Eq. (8). It follows that both $k_{i}^{\mathrm{el}}$ and $k_{i}^{\mathrm{ph}}$ are expected to vary with $x, y, z$ and $t$ rendering that behavior of thermal conductivity with anisotropy and inhomogeneity,

\section{Thermal transients in electromagnetics}

Thermal transient effects in ferromagnetic materials can also be treated by using the isoenergy theory. This can include temperature changes and energy dissipation. The details are in fact much less complicated that those encountered in the classical electromagnetic theory which involves a host of equations referred to Maxwell. They include the laws of Gauss, Ampere, Ohm and Faraday aside from the constitutive relations to include mechanical, electrical and magnetic effects.

In electromagnetics, there is the choice to prescribe a magnetic or electric field which give rise to displacement of the medium. To illustrate the simplicity of the procedure, the problem will be solved in the mathematical space of isoenergy density. It is imagined that the continuum is occupied by rectangular parallelpiped elements. Their size and position can vary from location to location and are determined according to the deformation and rotation time history, say caused by the magnetization of Permalloy 65 illustrated in [52]. Aside from the isoenergy density approach, there are no other known methods that could determine the nonequilibrium saturation characteristics of magnetization in addition to predicting the electromagnetic properties such as resistivity, permeability, etc., by theoretical means.

\subsection{Nonhomogeneous physical and thermal proper- ties}

The transient response of magnetization is responsible for the spatio-temporal changes of electromagnetic properties. This could be determined from a knowledge of the volume energy density function

$\frac{\mathrm{d} W}{\mathrm{~d} V}=\int h \mathrm{~d} b$.
In Eq. (22), $b$ and $h$ are the magnetic flux density and field intensity referred to the isoenergy density space. The problem is considered solvable since $f(e)$ in Eq. (12) depending only on the displacement gradients can be related to $b$ and $h$ in Eq. (12). The procedure for finding $b$ and $h$ will be illustrated and explained.

Let $(\mathrm{d} W / \mathrm{d} V, e)(h, e)$ and $(h, b)$ corresponds to an isoenergy density element at a given time in a system under consideration. With $e$ being the isostrain, a state $P_{1}$ in $(\mathrm{d} W / \mathrm{d} V, e)$ domain can be related to $P_{2}$ and $P_{3}$ in the respective $(h, e)$ and $(h, b)$ domains without ambiguity. A particular value of $b$ can thus be identified with $e$. What Fig. 9 illustrates is that $b$ and $h$ are basically known from $\mathrm{d} W / \mathrm{d} V$ for a given $e$. The magnetic flux density $B$ and field intensity $H$ in the physical plane are related to $b$ and $h$ through coordinate transformations.

Permeability. For a cylindrical conductor that possessed axisymmetry, the magnetic permeability coefficients $\mu_{r}$ and $\mu_{z}$ can be obtained as

$\mu_{r}=\frac{B_{r}}{H_{r}}, \quad \mu_{z}=\frac{B_{z}}{H_{z}}$,

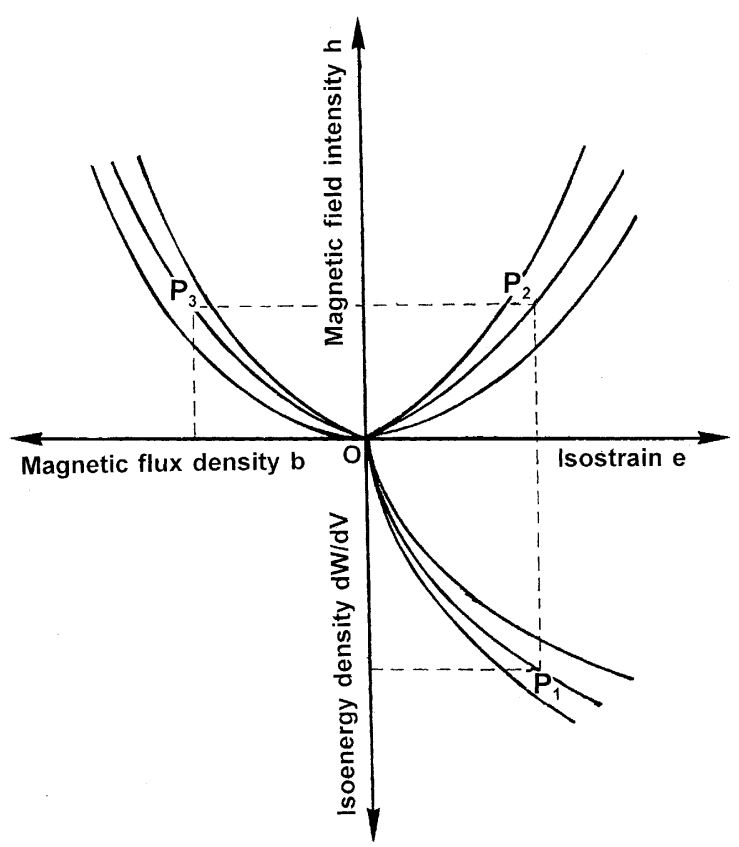

Fig. 9. Correspondence among the magnetic and isoenergy density quantities. 
since $B_{i}$ and $H_{i}(i=r, z)$ are found without knowing $\mu_{r}$ and $\mu_{z}$. The subscripts $r$ and $z$ refer, respectively, to the radial and axial directions of the cylindrical bar.

Resistivity electronic conductivity. Once $B$ is known, the magnetic flux $\Phi$ follows from

$\Phi=\int_{A} B \mathrm{~d} A$,

where $A$ is the surface area of the cylinder. Faraday's law gives the electric field intensities

$E_{r} \approx 0, \quad E_{\theta}=-\frac{1}{2 \pi r_{0}} \frac{\partial \Phi}{\partial t}$,

with $r_{0}$ being the radius of the cylinder. The electric current densities $J_{r}$ and $J_{\theta}$ can be estimated from Ampere's law:

$J_{r} \approx 0, \quad J_{\theta}=\frac{\partial H_{r}}{\partial z}-\frac{\partial H_{z}}{\partial r}$.

Since $J_{r}$ and $E_{r}$ are negligible, the resistivity $\mathscr{R}$ or electric conductivity $\mathscr{C}$ can be obtained directly from

$\mathscr{R}=\frac{E_{\theta}}{J_{\theta}}$.

Keep in mind that $\mathscr{R}$ can still depend on $r, z$ and $t$.

Dielectric permittivity. Since $J_{\theta}$ is known from $H_{r}$ and $H_{z}$ in Eq. (26) and from Eq. (25), the dielectric permittivity coefficient $\varepsilon$ can be extracted from the relation:

$J_{\theta}=\varepsilon \frac{\partial E_{\theta}}{\partial t}$.

Thermal conductivity. Following the definition of $k_{i}$ in Eq. (28), thermal conductivities $k_{r}$ and $k_{z}$ are given by

$k_{r}=C_{\Theta}\left(\frac{\mathrm{d} V}{\mathrm{~d} A}\right)_{r} \frac{\mathrm{d} x_{r}}{\mathrm{~d} t}, \quad k_{z}=C_{\Theta}\left(\frac{\mathrm{d} V}{\mathrm{~d} A}\right)_{z} \frac{\mathrm{d} x_{z}}{\mathrm{~d} t}$.

Anisotropy in addition to inhomogeneity are seen to prevail in the thermal conductivity coefficients given by Eq. (29).

\subsection{Magnetization of Permalloy 65 bar}

Consider a ferromagnetic cylindrical bar $2 \mathrm{~cm}$ in diameter and $16 \mathrm{~cm}$ in length to be surrounded by a coil which carries electric current. This is equivalent to a magnetic field with intensity $H$ which is simulated by a region $3.08 \mathrm{~cm}$ in diameter and $26 \mathrm{~cm}$ in length. This becomes a two-phase media problem as defined in Fig. 10. The Permalloy 65 composition consists of $45 \% N_{i}$ while the remainders are iron and impurities. The initial and maximum permeabilities are, respectively, $2.5 \times 10^{3}$ and $500 \times 10^{3} \mathrm{~Wb}^{2} / \mathrm{Nm}^{2}$. The average handbook value for saturation by induction is $13,300 \mathrm{G}$. It suffices to specify the global $H$ versus $B$ and $H$ versus $\epsilon$ (ordinary strain) data [53] although the specimen size is not reported. They are shown in Figs. 11(a) and (b). Spatio-temporal variations of the electromagnetic physical parameters for a Permalloy 65 cylinder engulfed by a uniform magnetic field are predicted. The averaged or global values agree surprisingly well with those in the handbook [53] obtained by test.

Field intensity and flux density. The variations of $B_{i}$ and $H_{i}(i=r, z)$ with $r, z$ and $t$ for each element in the Permalloy 65 cylindrical bar are predicted from the isoenergy density theory. Only their global averages denoted by $\left(B_{r}, H_{r}\right)$ and $\left(B_{z}, H_{z}\right)$ will be presented for discussion. Fig. 12 shows the time dependency of $B_{z}$ and $H_{z}$ in the axial direction. The effects of $B_{r}$ and $H_{r}$ in the radial direction are small and can be ignored. Predictions from the isoenergy density theory agreed well with the test data [53] despite the lack of information on the size of air gap used in [53]. It is known that the airgap distance between the specimen and primary coil can influence the data. The same conclusion applies to the difference between the $\left(B_{r}, H_{r}\right)$ and $(B, H)$ curves in Fig. 13.

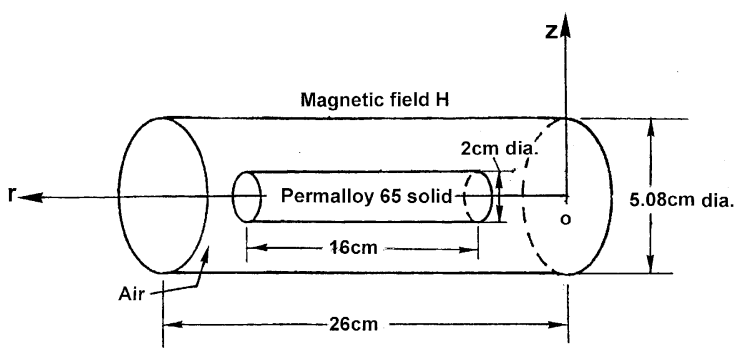

Fig. 10. Schematic of Permalloy $65 \mathrm{rod}$ in magnetic field. 

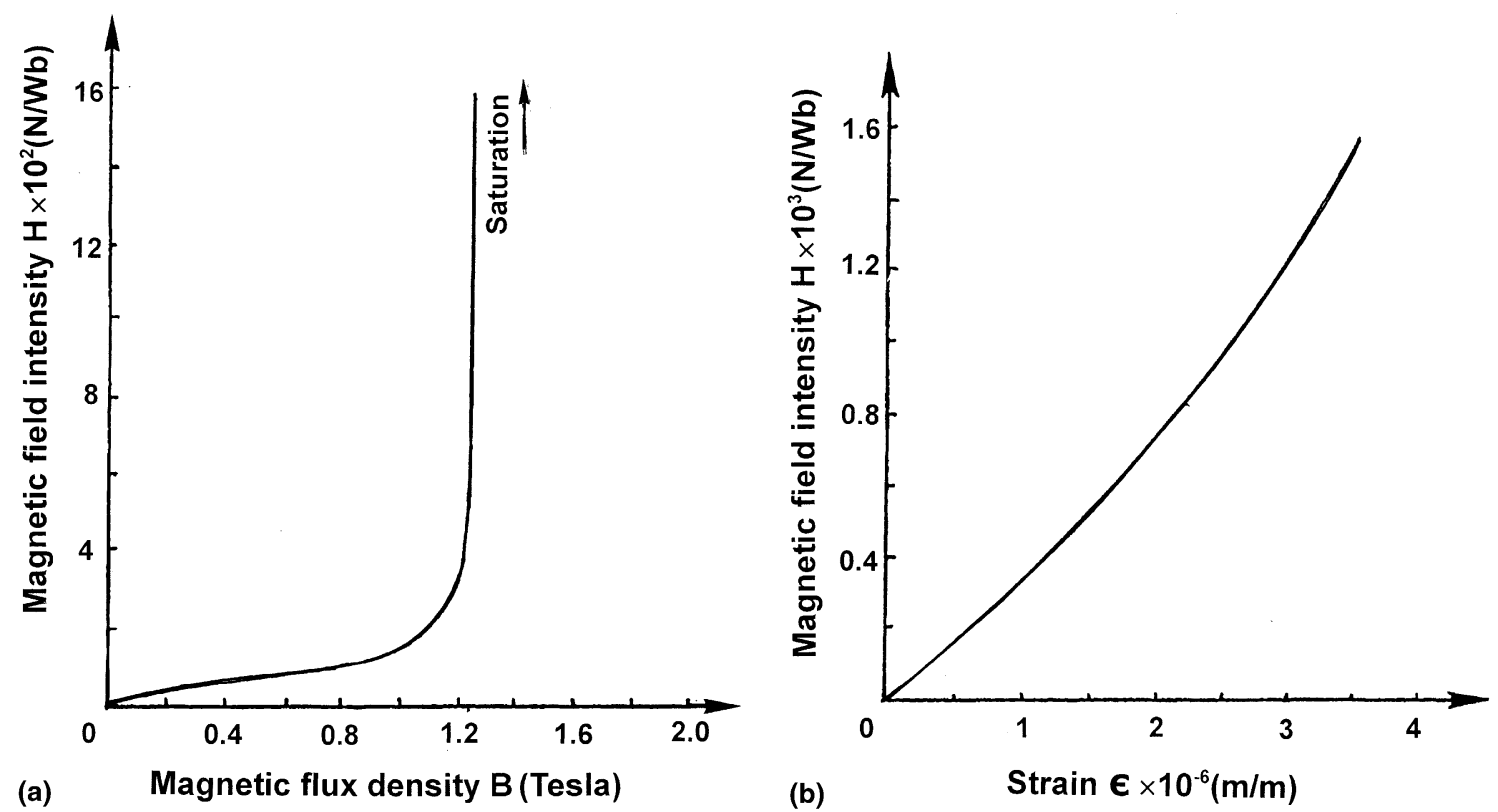

Fig. 11. Test data of magnetic field and flux density for Permalloy 65 [51]: (a) $H$ versus $B$; (b) $H$ versus $\epsilon$.

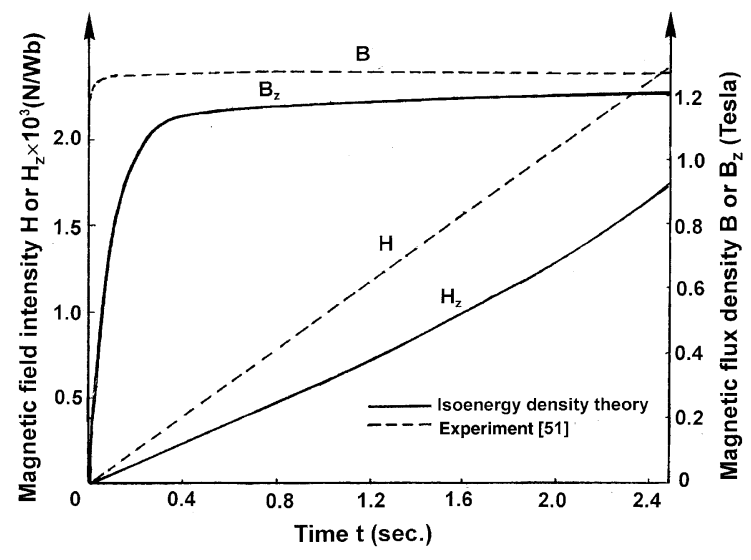

Fig. 12. Comparison of magnetic time response for Permalloy 65: prediction and experiment.

Time variations of permeability and resistivity. Displayed in Fig. 14 are the permeabilities $\mu_{r}, \mu_{z}$ as a function of $t$. Isotropy in magnetic permeability is exhibited since $\mu_{r}$ and $\mu_{z}$ are nearly equal for all time $t$ for Permalloy 65. Negligible difference in $\mu_{r}$ and $\mu_{z}$ is not unexpected since no preference in magnetic response due to material microsrtucture were introduced. A maximum value of $\mu$ (with

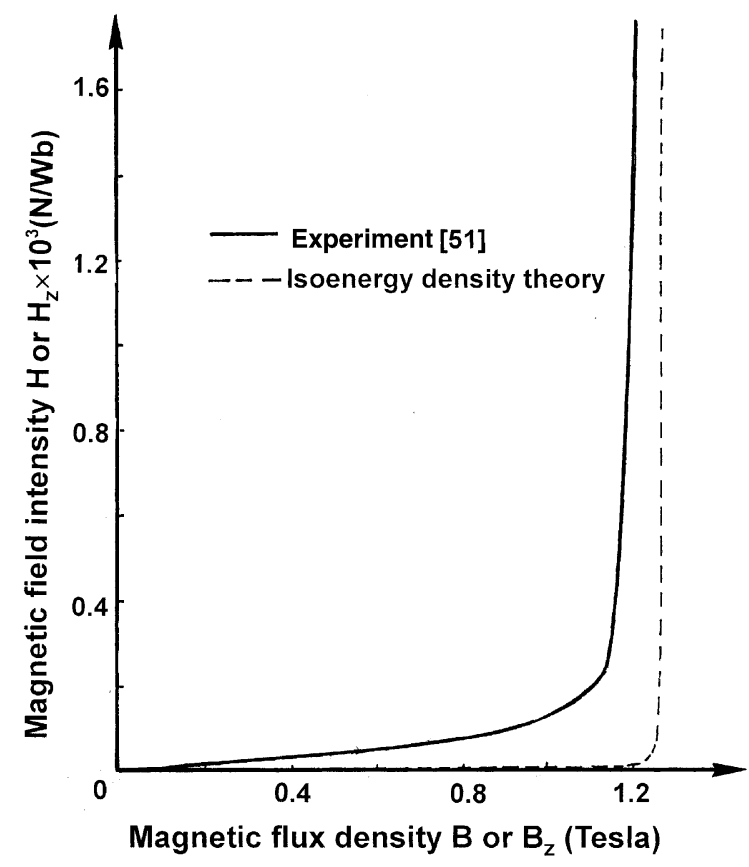

Fig. 13. Magnetization curve for Permalloy 65: prediction and experiment. 


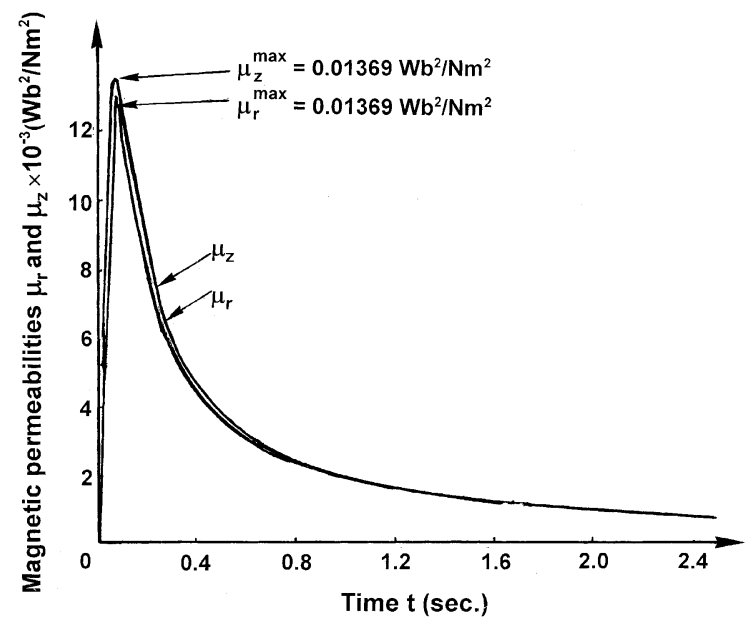

Fig. 14. Predicted axial and radial magnetic permeability for Permalloy 65.

reference to $B$ ) or $\mu^{\max }=5.97 \times 10^{-2} \mathrm{~W} \mathrm{~b}^{2} / \mathrm{Nm}^{2}$ was found in [54, p. 114]. This is within the same order of magnitude $\mu_{r}^{\max } \approx \mu_{z}^{\max }=1.3 \times 10^{-2}$ $\mathrm{W} \mathrm{b} / \mathrm{Nm}^{2}$ as predicted. Keep in mind that the magnetization characteristics could change appreciably if the heat treatment for two of the same ferromagnetic materials are varied.

Recall that the resistivity $\mathscr{R}$ can be determined from $E_{\theta} / J_{\theta}$ in Eq. (27) since $E_{r}$ and $J_{r}$ are negligibly small in comparison. The analytical predictions in Fig. 15 show that $E_{\theta}$ decays sharply with time

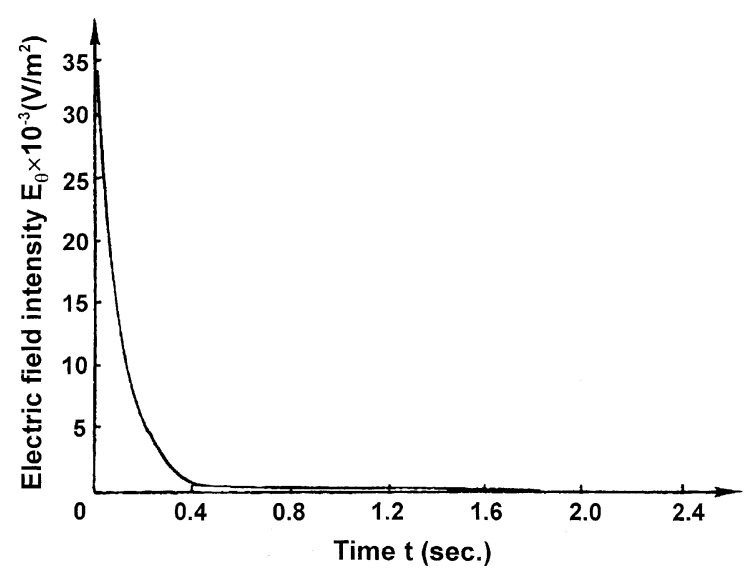

Fig. 15. Predicted overall average of electric current density versus time for Permalloy 65.

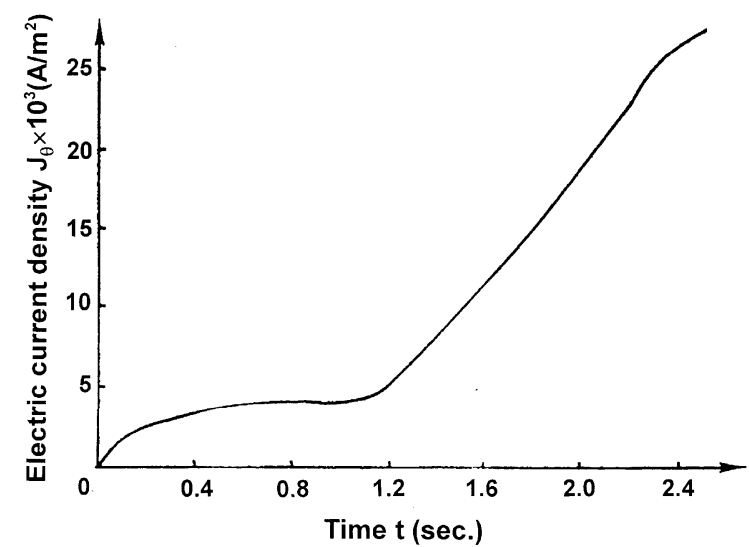

Fig. 16. Predicted overall average of electric field intensity as a function of time for Permalloy 65 .

while $J_{\theta}$ increases slowly with time at first and then more quickly, Fig. 16. Of particular interest is the time dependence of $\mathscr{R}$ in Fig. 17 where the resistivity dropped three orders of magnitude within a time span of $0.4 \mathrm{~s}$. That is $\mathscr{R}$ decreased from $2 \times 10^{-4} \Omega \mathrm{m}$ to the experimental values of 15 or $20 \times 10^{-8} \Omega \mathrm{m}$ in [53]. High initial resistance contributes to large energy loss.

A plot of the global dissipation energy density $\mathscr{D}$ against time is given in Fig. 18. For very small time $t$, the events correspond to those at lower scales. The curve is nearly flat before it rises rapidly. This corresponds to microscopic cooling

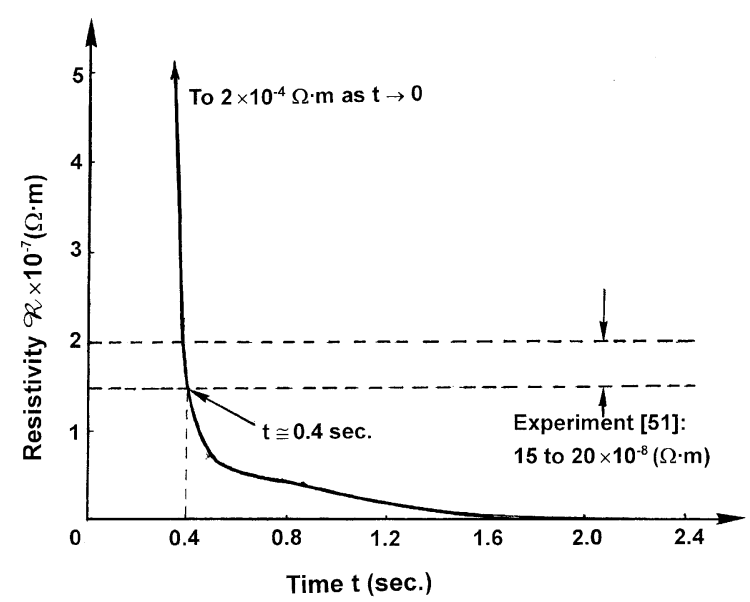

Fig. 17. Predicted time variation of resistivity for Permalloy 65. 


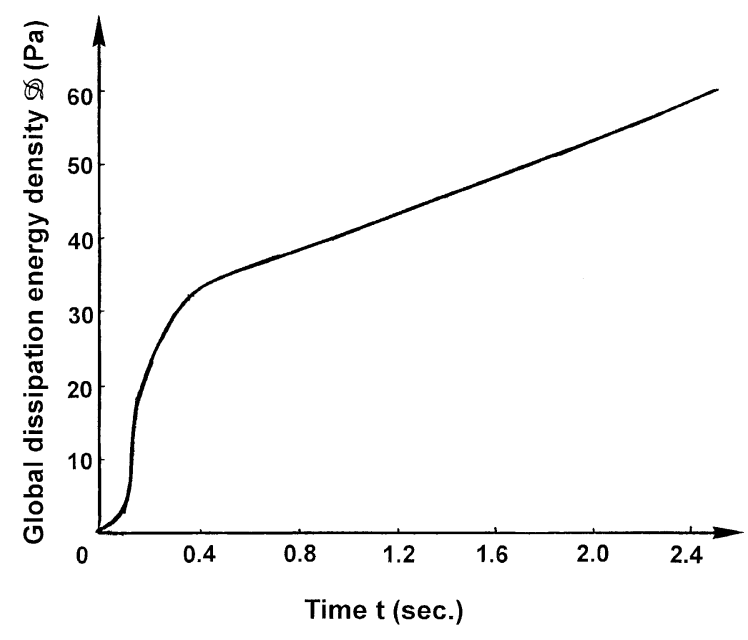

Fig. 18. Global dissipation energy density for Permalloy 65.

where the temperature would decrease below that of ambient. This will be identified with reversible domain displacement in magnetization being analogues to the linear elastic range in mechanical deformation. More on this will be discussed. Note from Fig. 18 that $\mathscr{D}$ is approximately $33.22 \mathrm{~Pa}$ at $t \approx 0.4 \mathrm{~s}$. For the remainder time span of $0.4-2.5 \mathrm{~s}$, an additional increase of $26.52 \mathrm{~Pa}$ is observed. This shows that about $56 \%$ of the dissipation energy occurs during an initial time period of approximately one-fifth of the total time span. Gross error could result from using the open literature data for electric resistivity [53] that assumes to be time independent. According to Fig. 17, $\mathscr{R} \approx 2.93 \times$ $10^{-6} \Omega \mathrm{m}$ at $t=0.25$ differs about one order of magnitude from the value reported in [53] for Permalloy 65. Such a discrepancy indicates that more attention should be given to the measurement of electric resistivity, particularly in the ways with which test data are reported. Also keep in mind that they are bulk properties of $\mu$ and $\mathscr{R}$. Properties of $B, H, \mu, \mathscr{R}$, etc., could deviate significantly from those mentioned earlier when the specimen size reduces to microns.

\subsection{Domain inhomogeneity}

One of the basic contributions of nonequillibrium thermoelectromagnetics is defining "domain inhomogeneity" based on $(\mathrm{d} V / \mathrm{d} A, \phi)$ in the system. The angle $\phi$ determines the direction of magnetic field $H$ as

$\phi=\tan ^{-1}\left|\frac{H_{z}}{H_{r}}\right|$

for the cylindrical bar system at hand. Depicted in Figs. 19(a) and (b) are change of element configuration from the dotted to the solid after application of a magnetic field in the $z$-direction that coincides with the longitudinal axis of the cylindrical bar. Magnetization can be identified with a

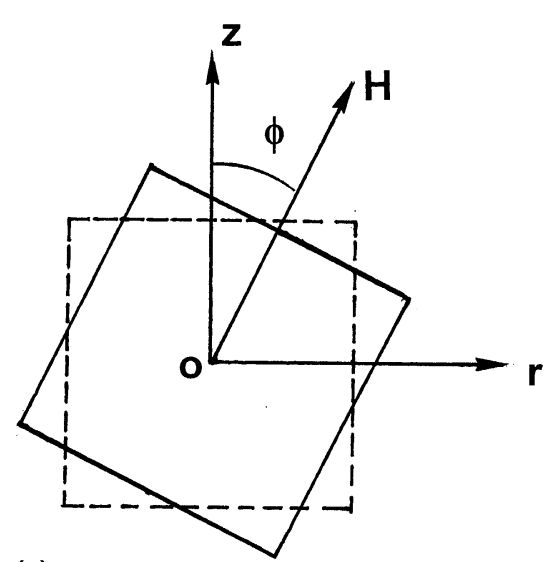

(a)

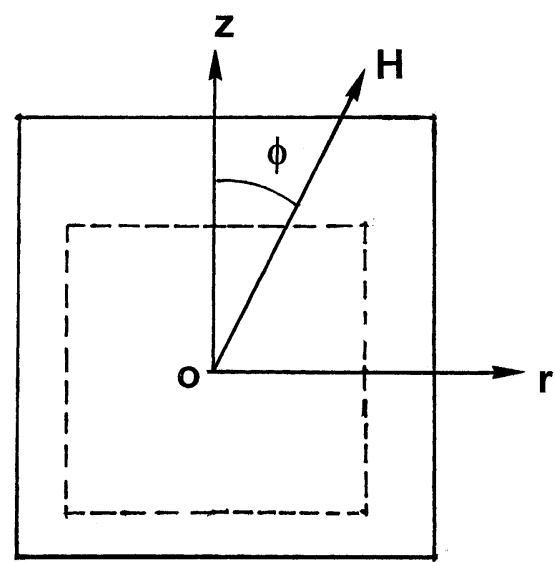

(b)

Fig. 19. Fundamental mechanisms of magnetization: domain displacement and rotation: (a) domain rotation (small $\mathrm{d} V / \mathrm{d} A$; large $\phi$ ); (b) domain displacement (large $\mathrm{d} V / \mathrm{d} A$; small $\phi$ ). 


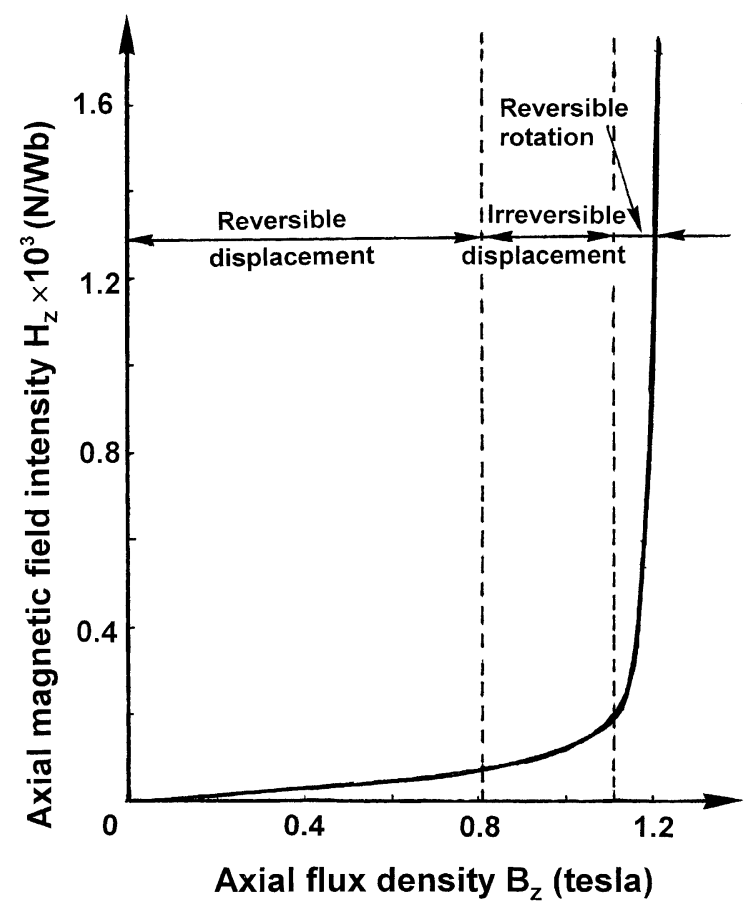

Fig. 20. Domain displacement and rotation identified on the magnetization curve predicted for Permalloy 65.

reversible and an irreversible part. Both of these parts can be related to domain displacement and domain rotation of the element as shown in Figs. 19(a) and (b), respectively. More specifically, the former corresponds to small $\mathrm{d} V / \mathrm{d} A$ and large $\phi$ while the latter to large $\mathrm{d} V / \mathrm{d} A$ and small $\phi$. A ranking system can thus be develop[ed to quantify the degree of inhomogeneity of the system. Three of the four mechanisms of magnetization are discussed in text books on physics; they consist of reversible domain displacement, reversible domain rotation and irreversible domain displacement. They are, identified with $\left(B_{z}, H_{z}\right)$ curve by the

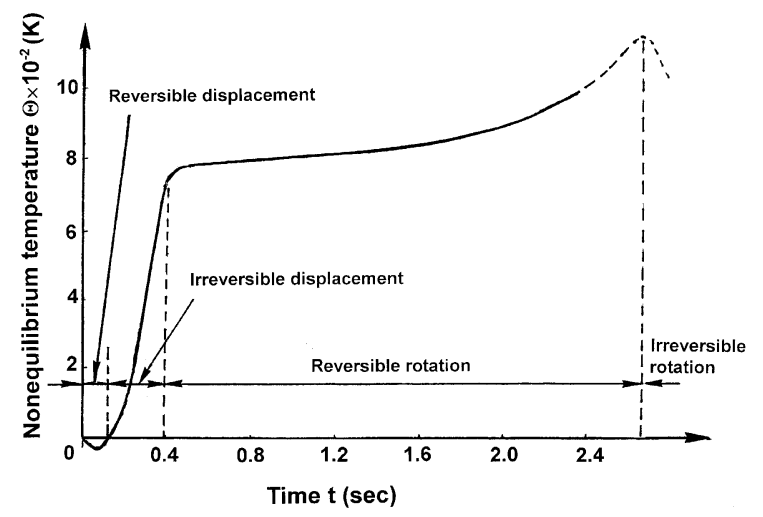

Fig. 21. Irreversible rotation identified with nonequilibrium time variation of temperature for Permalloy 65 .

segments shown in Fig. 20 for Permalloy 65. The irreversible domain rotation mode has only been speculated up to now. Validation of its existence was made possible from the nonequilibrium temperature curve in Fig. 21 which could not be found without the use of the isoenergy density theory. The final portion of the $\Theta$ curve where it drops sharply was anticipated as the final stage of damage. The corresponding time of about $2.65 \mathrm{~s}$ is estimated from Fig. 17, where $\mathscr{R} \rightarrow 0$. The oscillatory character of $\Theta$ with time in Fig. 21 is reminiscent of material damage at the different size scales as it was demonstrated in $[40,41]$ for the uniaxial tension and compression specimens. The temperature oscillation behavior was assessed quantitatively from the macro- to the microscale with the anticipation that further reduction in size scale would lead to thermal fluctuations at the atomic level.

Sixteen different degrees of domain inhomogeneity was classified in [52]; they are outlined in Table 5. An upward and download arrow refer, respectively, to relatively high and low value of the

Table 5

Classification of domain inhomogeneity

\begin{tabular}{llll}
\hline Magnitude & Domain & \\
\cline { 2 - 3 } & Rotation & Mixed & Displacement \\
\hline High & $(\mathrm{d} V / \mathrm{d} A \downarrow ; \phi \uparrow)$ & $(\mathrm{d} V / \mathrm{d} A \uparrow$ or $\downarrow ; \phi \uparrow$ or $\downarrow)$ & $(\mathrm{d} V / \mathrm{d} A \uparrow ; \phi \downarrow)$ \\
Medium & $(\mathrm{d} V / \mathrm{d} A \downarrow$ or $\downarrow ; \phi \uparrow$ or $\uparrow)$ & $(\mathrm{d} V / \mathrm{d} A \uparrow$ or $\uparrow ; \phi$ or $\uparrow)$ & $(\mathrm{d} V / \mathrm{d} A \uparrow$ or $\uparrow ; \phi$ or $\downarrow)$ \\
Low & $(\mathrm{d} V / \mathrm{d} A \downarrow \phi \uparrow)$ & $(\mathrm{d} V / \mathrm{d} A \uparrow$ or $; \phi \uparrow$ or $\vdots)$ & $(\mathrm{d} V / \mathrm{d} A \uparrow ; \phi \downarrow)$ \\
\hline
\end{tabular}


quantity next to it. Domain rotation corresponds to a downward arrow for $\mathrm{d} V / \mathrm{d} A$ and an upward arrow for $\phi$; the opposite applies to domain displacement. The two remaining combinations are applied to the mixed domain. The same interpretation holds for the dotted arrows except that they signify lower magnitude. Solid and dotted arrows could be combined to represent a mixture of magnitude for $\mathrm{d} V / \mathrm{d} A$ and $\phi$. Computations of $(\mathrm{d} V / \mathrm{d} A, \phi)$ have been made for Permalloy 65 [52]. Experimental observations should be carried out to check the scheme deviced to quantify magnetization inhomogeneity.

\subsection{Saturation}

The phenomenon of "saturation" is usually related to the intrinsic induction of $B$ approaching a finite limit as $H$ continues to increase. The behavior is exhibited by the $\left(B_{z}, H_{z}\right)$ or $(B, H)$ curves in Figs. 13 and 20 for Permalloy 65, where $H_{z}$ or $H$ increases sharply with little or no change in $B$. The open literature provides information only on global saturation, i.e., average characteristics of the elements in the specimen. Saturation of course would not take place homogeneously in a specimen. That is the $B-H$ response may differ locally, an assessment of which becomes more and more critical when the physical size of the system continuous to decrease. Linearity between $B$ and $H$ may not hold everywhere.

As the $B=B(H)$ relation may be used specifically in hightech applications, it is not sufficient just to know the average. Variations of $B$ and $H$ as a function of the space and time variables should be determined. Those factors that affect the electomagnetic properties of a given material should enter into the calculations for determining $B$ and $H$. They may include

- Chemical composition and crystal structure.

- Mechanical and heat treatment.

- Impurities such as \% carbon in iron.

- Internal plastic strain.

- Environment temperature.

Illustrated in Figs. 22-24 are some typical factors that could alter the behavior of a magnetization curve. Mechanical and heat treatments are shown in Fig. 22, while percentage of carbon in

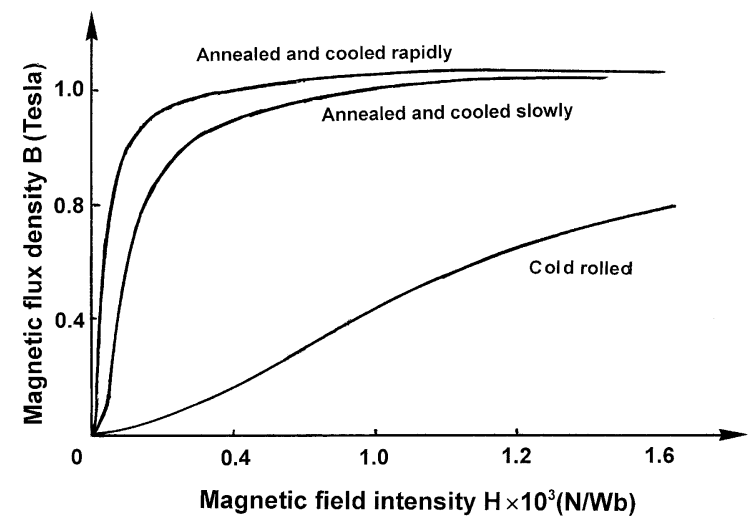

Fig. 22. Effect of mechanical and heat treatment of Permalloy 70 on magnetization curve [52].

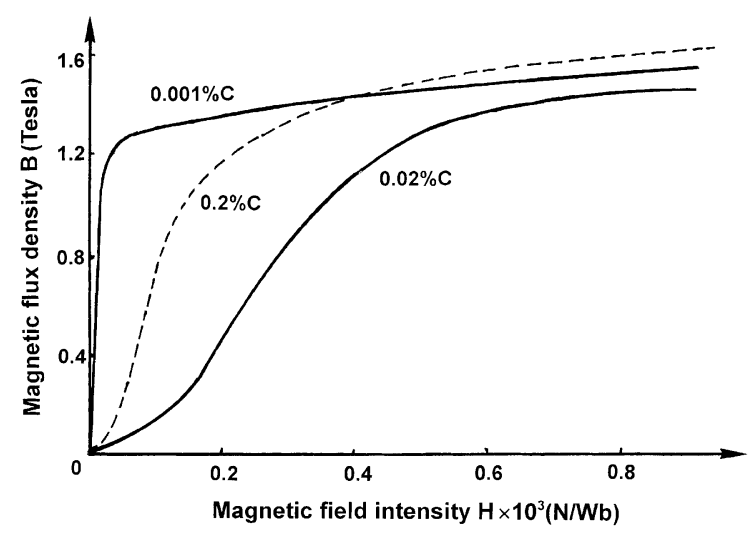

Fig. 23. Effect of impurities on magnetic properties of iron [52].

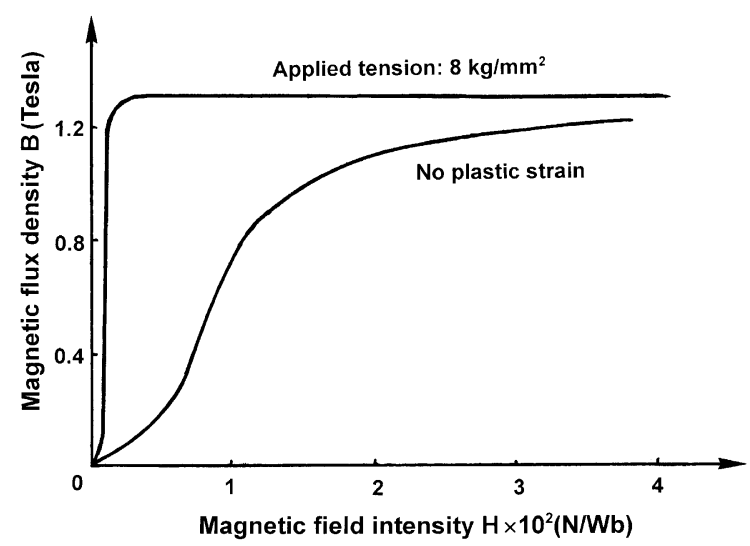

Fig. 24. Effect of plastic strain caused by tension for Peralloy 68 on magnetization curve. 
iron and initial plastic strain shown in Figs. 23 and 24 , respectively. Annealing reduction in $\%$ of carbon and plastic strain to sharpen the knee of the magnetic flux density curve. These effects are quite pronounced and could be assessed by the isoenergy density theory. More elaborate testing procedures should also be developed such that measurement variations due to the airgap distance between the specimen and primary coil and the others can be better controlled. Uncertainity should be minimized so as not to overshadow the $(B, H)$ variations caused by fabrication, heat treatment and other factors mentioned earlier.

\section{Paradigms in nonequilibrium thermomechanics}

The distinction of corpuscular and continuum models may seem to be far fetched from applications. It is not unthinkable to entertain the thought that an atom should have a definite size and shape when applying the molecular dynamics method. It may or may not be justified to model atoms as arrays of uniformly spaced points with interacting forces for estimating the movements of dislocations. More detail features of the atom, however, may be needed for the fabrication of macroscopic materials atom by atom. The circular appearance manifested by the high resolution electron microscope may simply represent the smeared motion of subatomic particles not necessary equal in size. On a more refined size scale, the continuum model may be a better representation of these particles. Even though the truth may never be known, there is the need to put forward a model that accounts for a wide variety of phenomena successfully. To emphasize is that there exists several individual effects to which diverse causes can be adjusted one to each. On the other hand, it is not so easy to adjust one and the same model to several effects, unless it be the one that possesses sufficient generality regardless of whether it adopts the corpuscular, continuum or mixed view. Conjectural character of scientific hypotheses cannot be avoided.

In what follows, the isoenergy density theory will be used to simulate a variety of physical processes that are nonequilibrium and nonhomogeneous in character. The rate change of volume with surface $\mathrm{d} V / \mathrm{d} A$ must necessarily remain finite for explaining nonequilibrium phenomena. The quantity $\mathrm{d} V / \mathrm{d} A$ is responsible for the existence of Brownian motion where thermal and mechanical effects are coupled.

\subsection{Cooling and heating}

When an ordinary uniaxial strip specimen is stretched in an open system, the temperatures within the specimen are not uniform and remain below ambient for sometime that is contrary to the general notion of the text book. The data in Figs. 25(a) and (b) illustrate this nonequilibrium behavior for the SAFC-40R steel specimen [36].
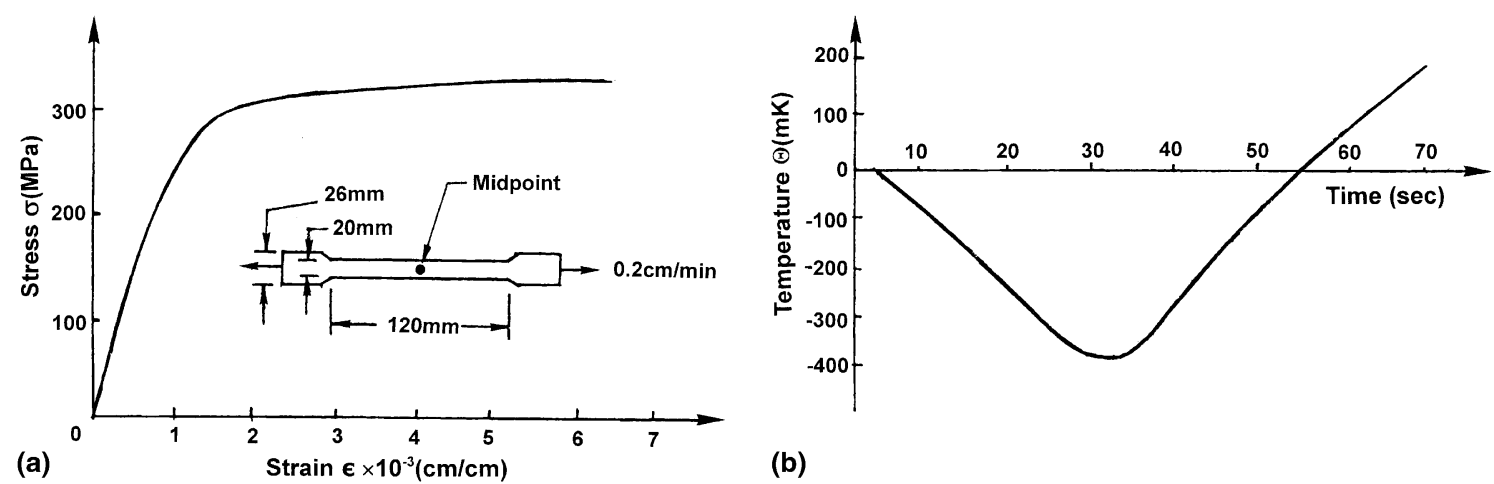

Fig. 25. Mid-point stress-strain and temperature for SAFC-40R steel strip in tension [34]: (a) mid-point stress and strain; (b) temperature at mid-point. 
The cross-head of the specimen is approximately $0.2 \mathrm{~cm} / \mathrm{min}$. Fig. 25(a) gives the stress-strain curve with the corresponding temperature time history in Fig. 25(b). For a significant portion of the stressstrain history (nearly $2 / 3$ or $55 \mathrm{~s}$ ) the specimen temperature was below ambient. The specimen heats up very rapidly prior to fracture, an event that occurs within the terminating portion of the stress-strain curve. The major portion of the $(\sigma, \epsilon)$ curve experiences nonequilibrium. This behavior is load rate dependent.

Fig. 26 shows the temperature-time variations for the 6061-T6 aluminum strip, the dimensions of which are given in Fig. 25(a). The heat displacement rates differ by one order of magnitude as shown. As the loading rate is decreased, the time for the specimen temperature to return ambient is increased from 25 to $195 \mathrm{~s}$, a difference of about $2.83 \mathrm{~min}$ where the trough depth of the temperature curve is decreased. This feature is not insignificant. It offers insights into the approachment of an adiabatic process as the slow loading rate curve flattens out to the horizontal axis and of a reversible isothermal process as the fast loading rate curve straightens to the vertical axis. In general, the behavior of an uniaxial stress-strain is nonequilibrium in character.

Fluctuation on the nonequilibrium temperature curve can also yield information with reference to material phase transformation. Such a feature was detected near the crack tip of a 1020 steel compact

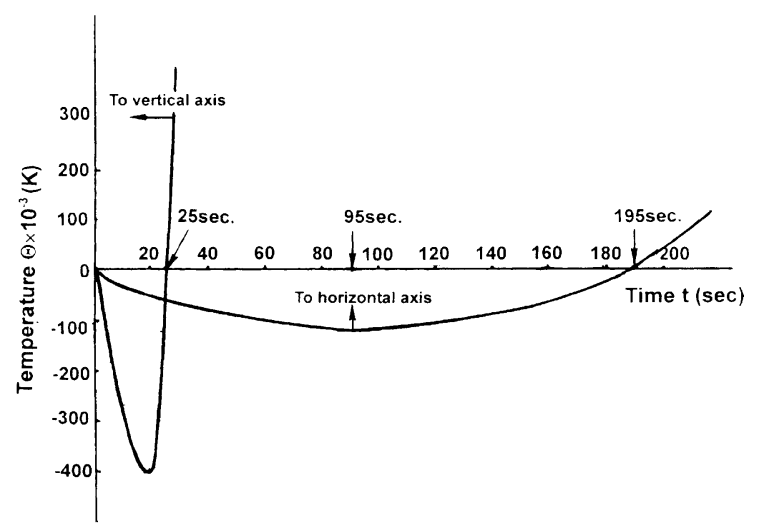

Fig. 26. Influence of loading rate on 6061-T6 aluminum strip in tension [35]. tension specimen [35] loaded by a rising load at a rate of $0.508 \mathrm{~cm} / \mathrm{min}$. Experiments were also made to check this behavior as displayed in Fig. 27. The local temperature fluctuation can be identified with curvature change on the $\mathscr{H}$-function versus time curve in Fig. 28. The $\mathscr{H}$-function as defined in the isoenergy density theory [15] is analogous to the entropy function in classical thermodynamics except that the former applies to nonequilibrium process (open system) and the latter to equilibrium process (closed system). In addition, $\mathscr{H}$ could be negative (order) and positive (disorder):

$\mathrm{d} \mathscr{H}=-\frac{\mathrm{d} \mathscr{D}}{\Theta}$.

Negative sign designates work done on the system. Variations of $\mathscr{H}$ with time $t$ for the problem in [35] are shown in Fig. 28. The local fluctuation $\mathscr{H}_{1}\left(t_{1}\right)$ corresponds to a small region approximately 0.1 $\mathrm{cm}$ ahead of the crack tip. Heating portion of the $\left(\mathscr{H}_{1}, t_{1}\right)$ curve starting at $t_{1}=42 \mathrm{~s}$ is given by

$\mathscr{H}_{1}=150.11 \log \left(\frac{t_{1}}{42}\right)$,

where

$\mathscr{H}=\mathscr{H}_{1}+0.538 \mathrm{MPa} / \mathrm{K}$.

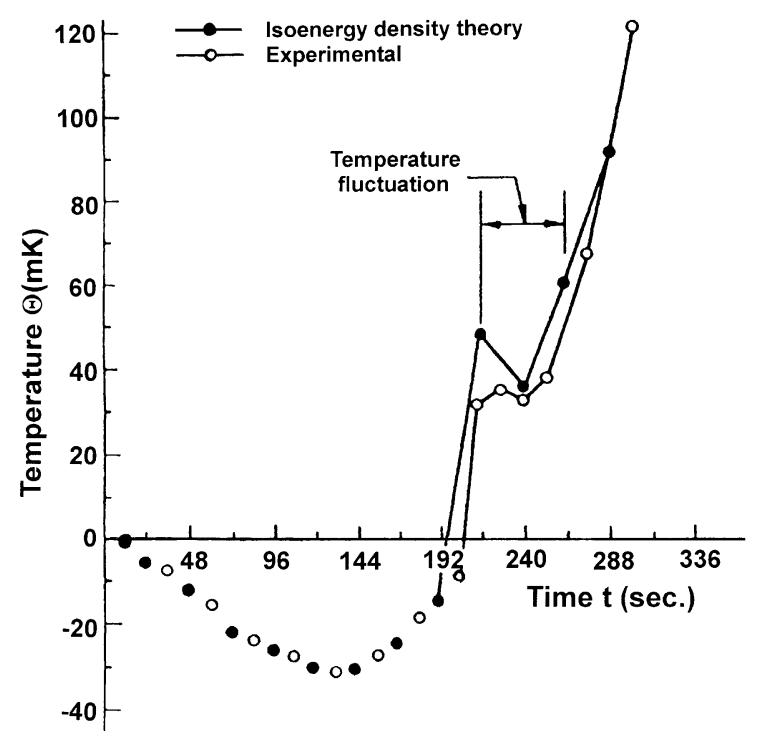

Fig. 27. Secondary temperature fluctuation ahead of crack in 1020 steel compact tension specimen. 


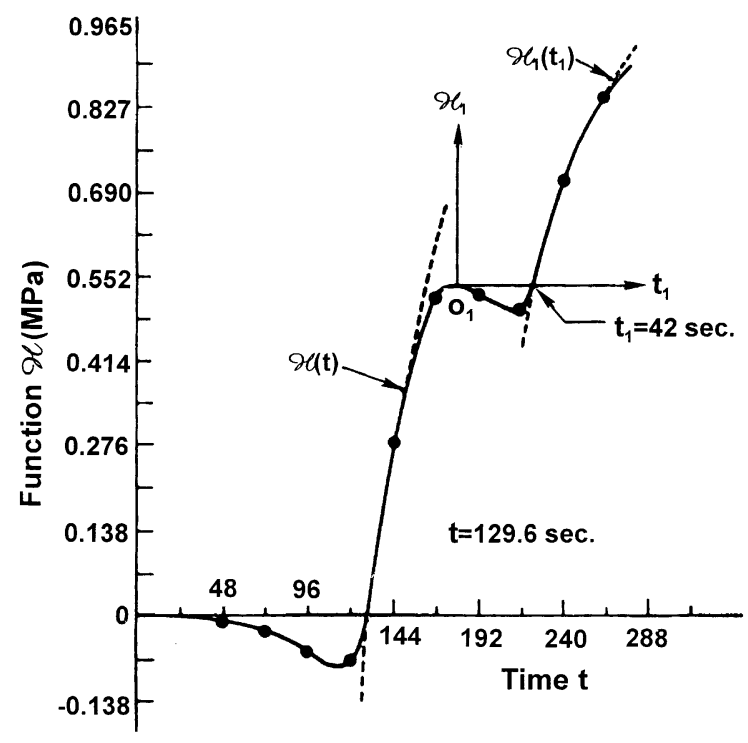

Fig. 28. Time history of $\mathscr{H}$-function ahead of crack in compact tension specimen [33].

The main portion of the heating curve is

$\mathscr{H}=820.3 \log \left(\frac{t}{129.6}\right)$.

At $t=129.6 \mathrm{~s}$ a reversal in heat transfer takes place. That is when heat is released from the cracked specimen to its surrounding as the material is damaged progressively. A second temperature drop is found to occur at $\mathscr{H}=0.538 \mathrm{MPa} / \mathrm{K}$ and $t=178.8 \mathrm{~s}$. This may be interpreted as a reassessment of order and disorder with reference to the $\mathscr{H}_{1}$ and $t_{1}$ system. The reassessment corresponds to phase transformation in the material microstructure. This was indeed observed on the fractured surface near the crack tip [35].

The concept of damage free zones at the different scale levels [10] was originated from the electron microscopic studies at the Oak Ridge National Laboratory [13] that the emission of dislocation stopped short at a small but finite distance ahead of a crack that is beyond the resolution needed to see dislocations. Hence, the connotation "damage free" has to be qualified with reference to size scale such as macrodamage or microdamage. For the same 1020 steel compact tension specimen analyzed in [35], the dissipation energy density function $\mathscr{D}$ is obtained and shown in Fig. 29 as a function of time. Within a local region with radius $4.75 \times 10^{-2} \mathrm{~cm}$ at time $t=80$ $180 \mathrm{~s}$, no energy dissipation could be detected at the macroscopic scale. This translates to the absence of damage and hence the "macrodamage free" zone description in Fig. 29. The analysis in [35] can be repeated to show that similar feature would prevail at the atomic and microscopic scale, Fig. 30. Dislocation free, microdamage free, and macrodamage free zones would correspond to linear distances of $10^{-8}, 10^{-4}$ and $10^{-2} \mathrm{~cm}$, respectively. These zones in fact refer to the limiting scale of observation.

A more general interpretation of $\Theta(t)$ variations have been given in $[40,41]$ for the tension and compression of 2024-T3 aluminum bars displaced at $2.12 \times 10^{-2} \mathrm{~mm} / \mathrm{s}$. Results in Fig. 31 for tensile bar demonstrate that size and time $t$ interact at the different scales. Cooling and heating tend to alternate with changes in size and time. The dotted portions of the curve were speculated while the solid portions were found by experiments. In view

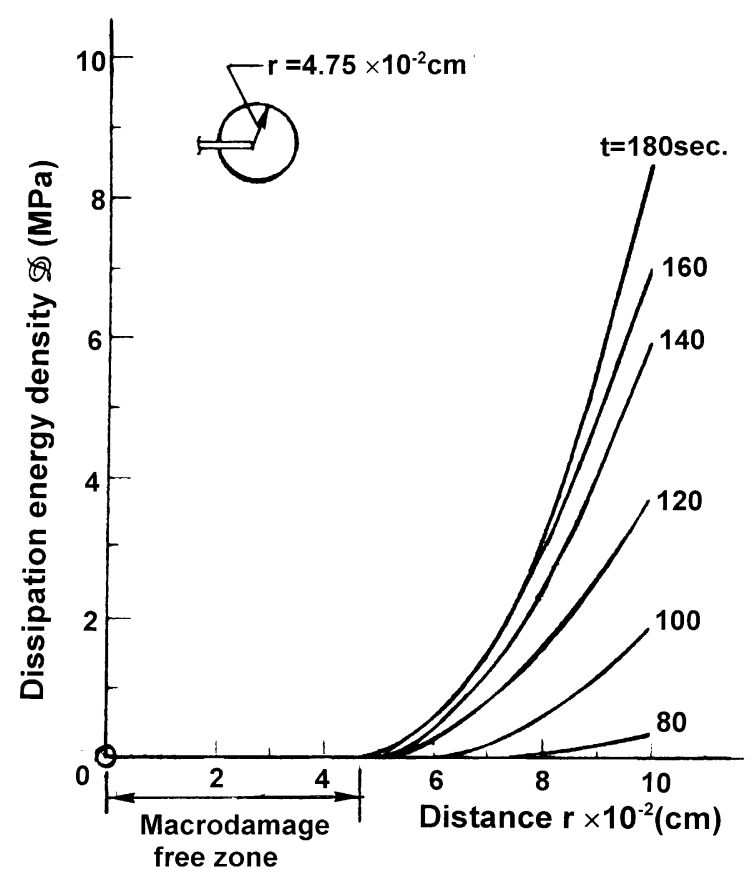

Fig. 29. Estimated macrodamage free zone for 1020 steel compact tension specimen at $0.508 \mathrm{~cm} / \mathrm{min}$. 


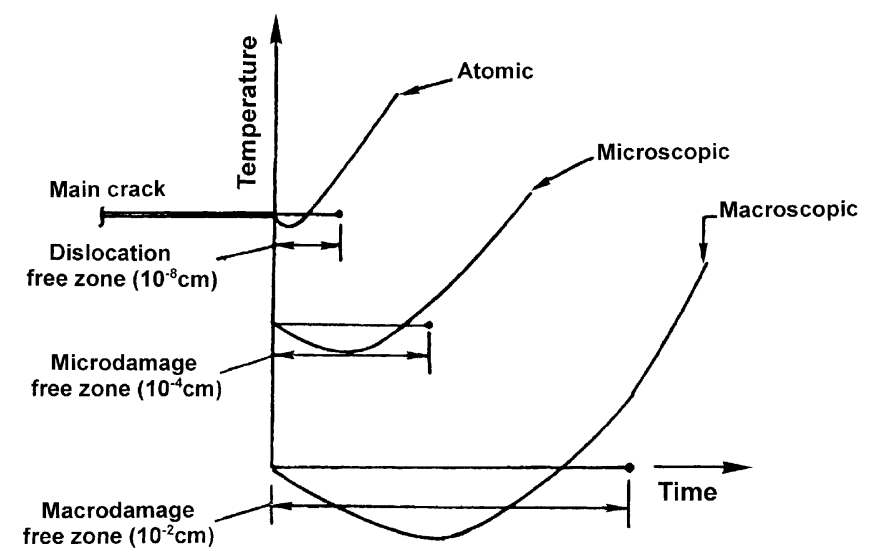

Fig. 30. Scaling of apparent damage free zones: cooling/heating.

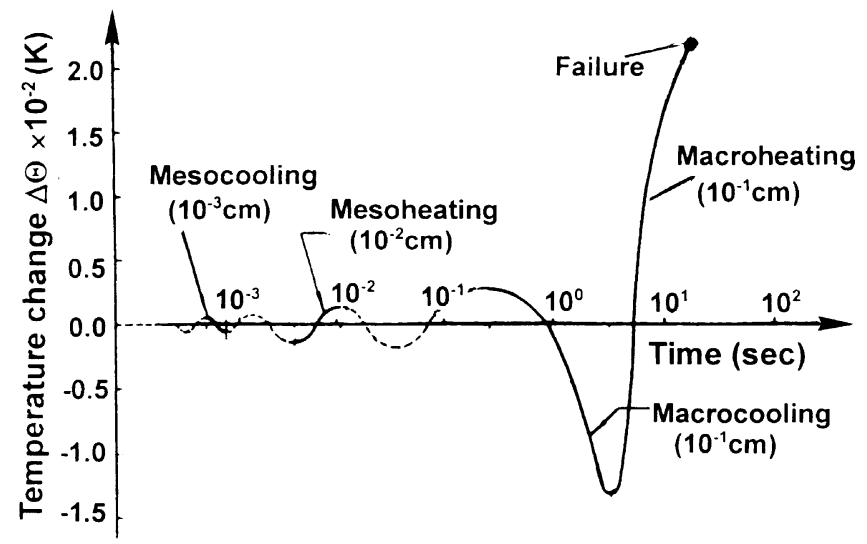

Fig. 31. Temperature-time history for 2024-T3 aluminum bar in tension at the macro- and mesoscale.

of the relation between $\mathrm{d} \Theta / \Theta$ and $\mathrm{d} V / \mathrm{d} A$ in Eq. (1), cooling and heating are also connected with dilatation and distortion of the material elements [55].

\subsection{Hypervelocity impact}

When a tungsten penetrator hits an aluminum target at $9000 \mathrm{~m} / \mathrm{s}$, both the penetrator and target will be severely damaged by fragmentation, fracture and phase transformation of the material microstructure where the solid may transform to liquid and/or gas nonuniformly throughout the system. The sequence of nonequilibrium damage states were traced in nanoseconds for a penetrator
$1.4 \mathrm{~cm}$ wide and $5 \mathrm{~cm}$ long and a 5083 aluminum plate $2 \mathrm{~cm}$ thick and $12 \mathrm{~cm}$ wide. Refer to [42] for details.

Within approximately $10-15 \mathrm{~ns}$, the local strain rate in the target increased to $10^{4} \mathrm{~s}^{-1}$ for the solid phase and $10^{5} \mathrm{~s}^{-1}$ for the liquid phase. Fig. 32 shows that at $t \approx 10 \mathrm{~ns}$, damage is confined to the corner region of the penetrator and target, a solid debris is detached from the target, ejecting from the system at a $70^{\circ}$ angle with a velocity of $74.09 \mathrm{~m} / \mathrm{s}$. A more refined analysis showed that a liquid layer has already been developed at the contact, Fig. 33. The estimate is that the liquid layer appeared near $t \approx 4 \mathrm{~ns}$, where $(\mathrm{d} \mathscr{D} / \mathrm{d} e)_{\mathrm{m}}=$ $25.71(\mathrm{MPa})$ which is beyond the critical value of 


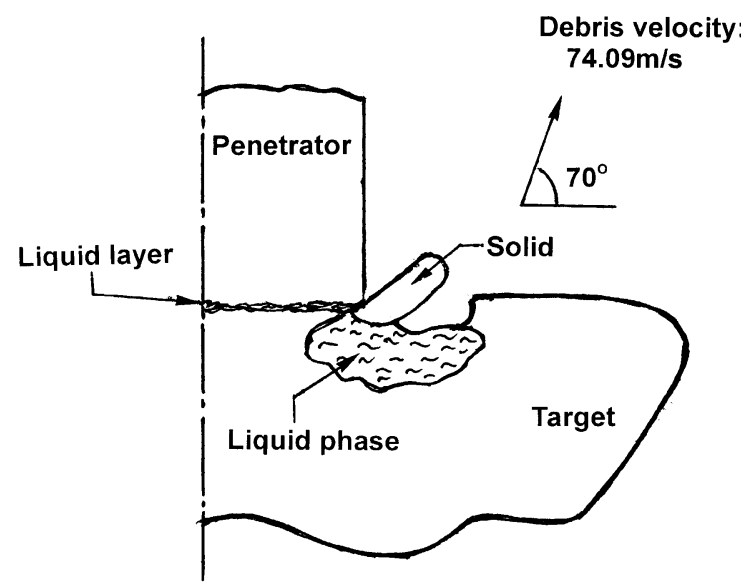

Fig. 32. Local damage at $t=10 \mathrm{~ns}$.

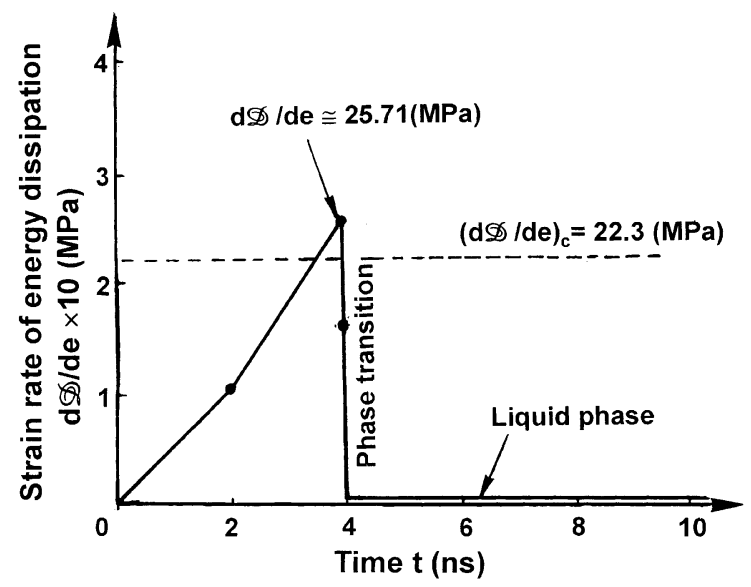

Fig. 33. Strain rate of energy dissipation as function of time at the contact.

22.3 MPa for aluminium to undergo phase transformation from solid to liquid. The value for tungsten is $96 \mathrm{MPa}$. The quantity $\mathrm{d} \mathscr{D} / \mathrm{d} e$ is associated with the latent heat in classical thermodynamics for determining the phase transformation of solid to liquid and gas except that $\mathrm{d} \mathscr{D} / \mathrm{d} e$ applies locally to transient conditions. Only when $\mathscr{D} \rightarrow Q$ then the following relation would apply

$\frac{\mathrm{d} \mathscr{D}}{\mathrm{d} e} \rightarrow \frac{\mathrm{d} Q}{\mathrm{~d} V}=T \frac{\mathrm{d} S}{\mathrm{~d} V}$.

In Eq. (35), $\mathrm{d} Q$ is the heat required per unit mass of substance in changing the thermodynamic state from the temperature $T$ to $T+\mathrm{d} T$ and $\mathrm{d} V$ is the

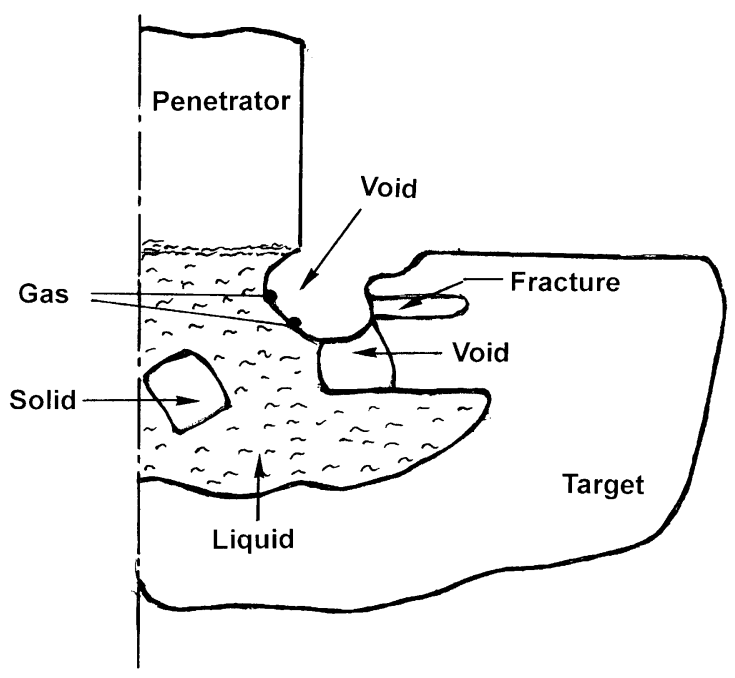

Fig. 34. Damage pattern at $t=15 \mathrm{~ns}$.

change of volume. The corresponding change in entropy is $\mathrm{d} S$. A small time interval $\mathrm{d} t$ is required to develop the liquid layer where slippage would take place.

At $t \approx 15 \mathrm{~ns}$, the local pressure and temperature next to the penetrator/target contact are so intense that the two small liquid regions $0.072 \mathrm{~mm} \times$ $0.191 \mathrm{~mm}$ and $0.143 \mathrm{~mm} \times 0.358 \mathrm{~mm}$ transform into gas. They are represented by the two solid dots in Fig. 34. The damage pattern for each time interval however small can be evaluated and displayed. Moving to $t=280 \mathrm{~ns}$, the solid island in Fig. 34 has now disappeared and transformed into liquid. Two large regions of vaporized liquid appear; they are indicated as "gas" in Fig. 35. The penetrator fails by fracture and ejection of debris continue to occur. The analysis could continue to predict disk-like spread of liquid as it is compressed by the penetrator. Sizable distortion and deformation are expected to form a crater. The computational effort should be less of a burden now that more efficient numerical algorithms and large-scale computing facilities are available.

\subsection{Electromagnetic launchers}

Rail guns and magnetic levitation vehicles discharge highly intensified energy much of which is dissipated a unuseful work. If it was not for the 


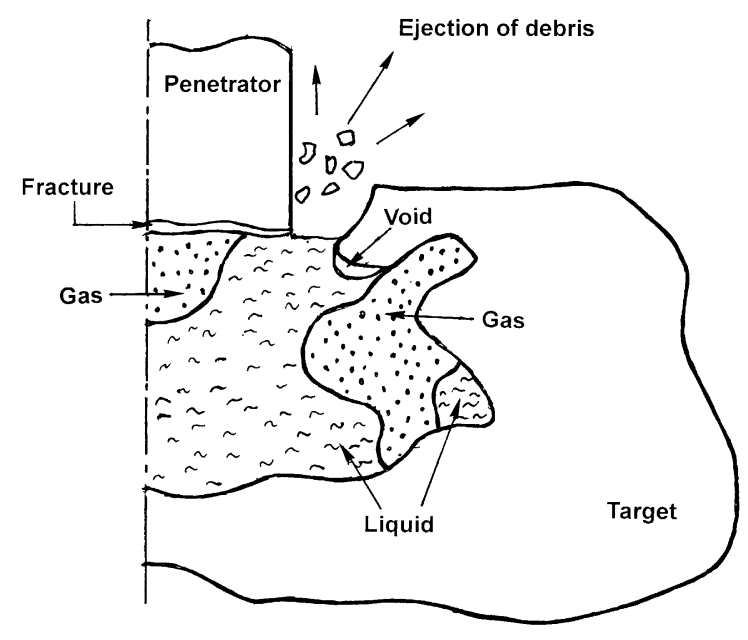

Fig. 35. Hypervelocity impact damage of projectile/target at $t=280 \mathrm{~ns}$.

advancement in superconductivity, many of the high-energy release operations would be rendered unpractical because of low efficiency. Control of energy dissipation characteristics appear to be a major factor in design. A typical $70 \mathrm{~kJ}$ railgun system [56] has been investigated [58] by evaluating the dissipation energy density quantity $\mathscr{D}$ in Eq. (1). In this way, the nonequilibrium temperature fluctuations in the railgun system can be estimated and checked experimentally.

A major design objective of the railgun is to accelerate projectiles up to $12-15 \mathrm{~km} / \mathrm{s}$. Large quantity of energy has to be stored and released within a very short period of time. This can be accomplished by using a magnetic flux compression current generator (MFCG) with a capacitor bank providing $600 \mathrm{~kJ}$ at $20 \mathrm{kV}$ within $40 \mathrm{~ms}$. A set of empirical data for the railgun current $I$ as a function of $t$ is given in Fig. 36. The time and current for detonation and discharge correspond, respectively, to $50 \mu$ s and $2 \times 10^{5} \mathrm{~A}$ (ampere) for the former and $3.56 \times 10^{-4} \mathrm{~s}$ and $5.75 \times 10^{5} \mathrm{~A}$ for the latter. Projectile displacements and velocities can be measured using X-ray shadow graph.

Making use of the data [57,58] for the equivalent current $I_{\text {eq }}$ and equivalent resistance $R_{\mathrm{eq}}$ in the rails, the energy transferred to the rails can be estimated as

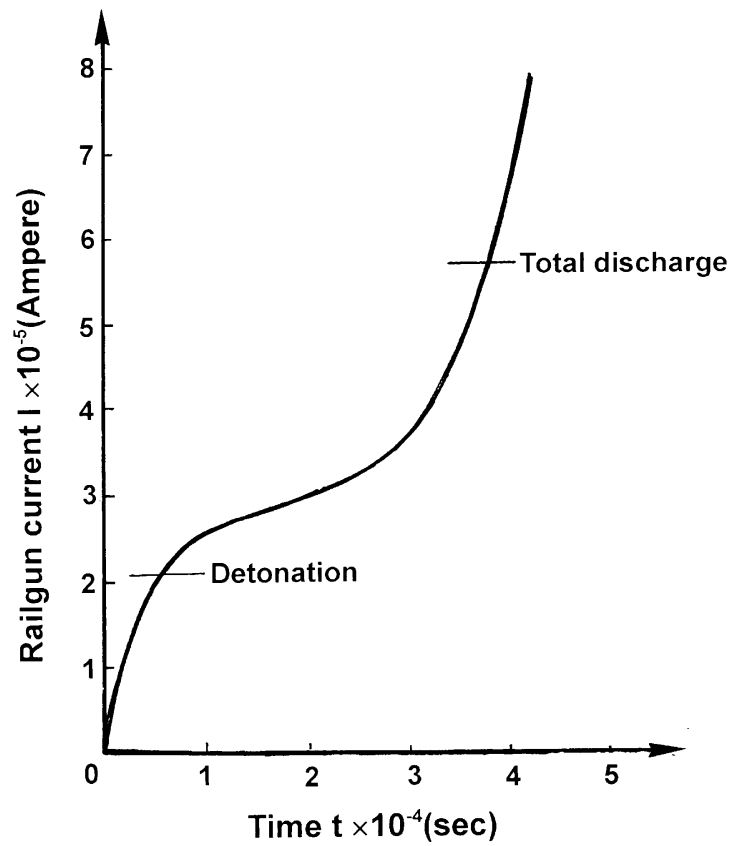

Fig. 36. Test data on railgun current versus time ( $70 \mathrm{~kJ}$ bank).

$U=\int_{0}^{t} R_{\mathrm{eq}} I_{\mathrm{eq}}^{2} \mathrm{~d} t$

It follows that the volume energy density $\mathrm{d} W / \mathrm{d} V$ in Eq. (2) can be obtained as $U$ in Eq. (36) divided by the rail volume given by $0.9 \mathrm{~m} \times 0.019 \mathrm{~m} \times$ $0.019 \mathrm{~m}$ for each rail. Plotted in Fig. 37 is the increase in $\mathrm{d} W / \mathrm{d} V$ as a function of time. It reached the level $30 \mathrm{MN} / \mathrm{m}^{2}$ (or MPa) at the end of $800 \mu \mathrm{s}$. Moreover, time variations of the magnetic intensity $H$ can be obtained from

$H=\frac{I}{\Delta y}$,

in which $I$ is the railgun current and $\Delta y=12.5 \times$ $10^{-3} \mathrm{~m}$ is the gap between the rails. Making use of the data in Fig. 36, Eq. (37) can be solved for $H$ in terms of time. The results in Figs. 37 and 38 can be combined to yield $\mathrm{d} W / \mathrm{d} V$ as a function of the magnetic intensity $H$. Graphical representation of the results can be found in Fig. 39. Once $\mathrm{d} W / \mathrm{d} V$ and $H$ are known, the magnetic flux density $B$ follows from 


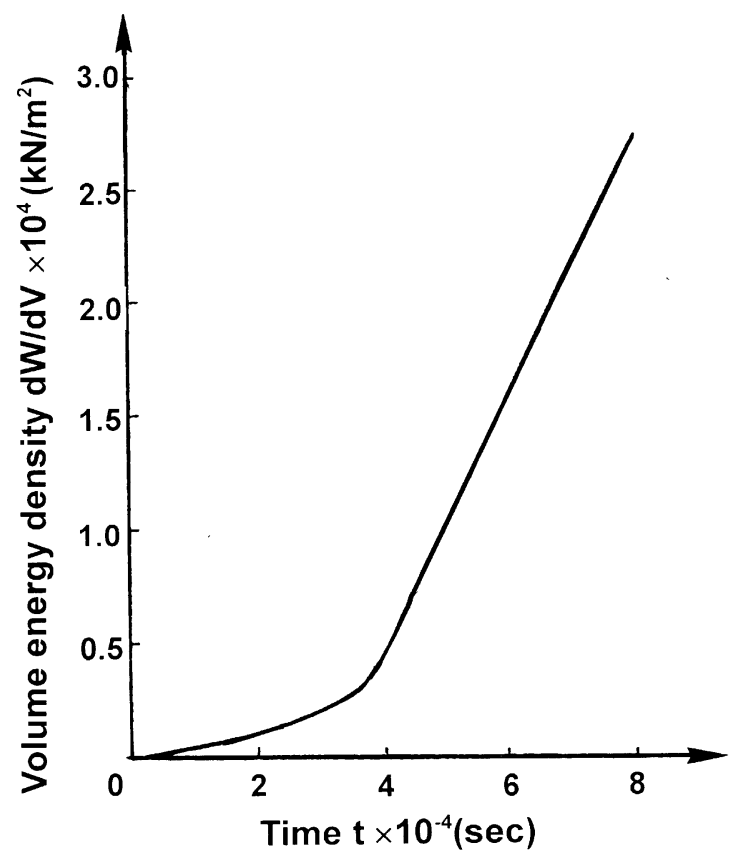

Fig. 37. Time variations of volume energy density for a $70 \mathrm{~kJ}$ capacitor bank system.

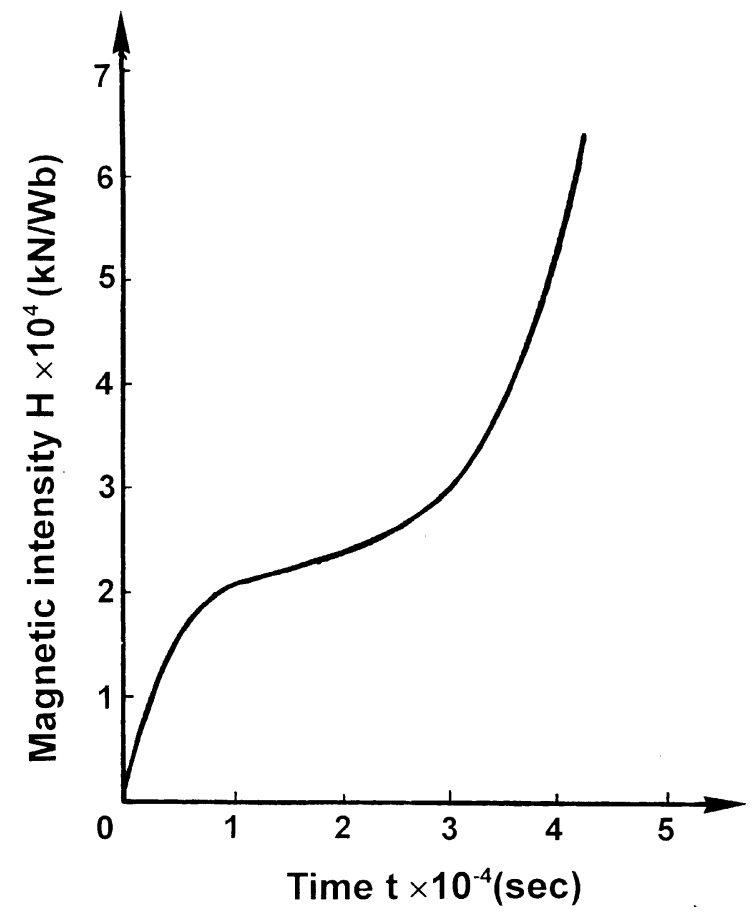

Fig. 38. Variations of magnetic intensity with time $(70 \mathrm{~kJ}$ bank).

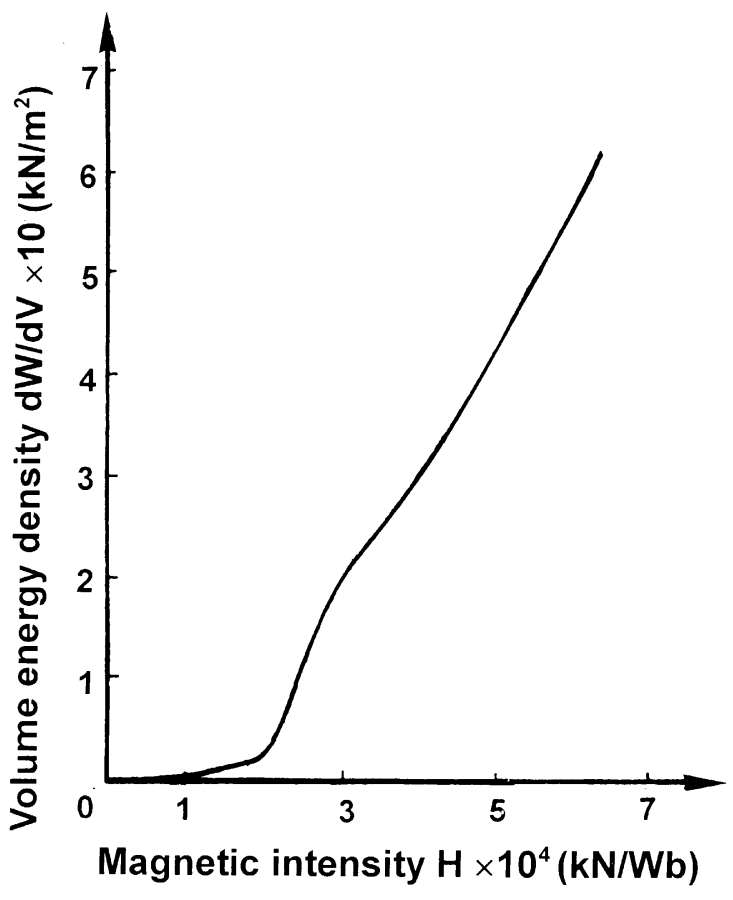

Fig. 39. Variations of volume energy density with magnetic intensity for a $70 \mathrm{~kJ}$ capacity bank railgun system.

$\frac{\mathrm{d} W}{\mathrm{~d} V}=\int H \mathrm{~d} B$

Eq. (38) is essentially Eq. (22) in which $h$ and $b$ are referred to the mathematical space of isoenergy density. This gives the $(B, H)$ curve behavior for a typical railgun system as shown in Fig. 40. At detonation which corresponds to $5 \mathrm{~ms}$, the slope of the $(B, H)$ curve increases dramatically. This corresponds to $B=0.4 \mathrm{~T}$ and $H=20 \mathrm{MN} / \mathrm{Wb}$.

Knowing the acceleration and mass of the projectile $(2.9 \mathrm{~g})$, the work done on the projectile as a function of time can be determined. The results are given in Fig. 41. The curve levels off a 10 $\mathrm{kJ}$ as the useful work. Based on the input of $70 \mathrm{~kJ}$, the efficiency is only about $14.29 \%$. This is evidence of the fact that much of the energy has been absorbed by the railgun structure components which are known to inflict several damage after a single firing. Based on a $15 \%$ efficiency, the dissipation energy density $\mathscr{D}(t)$ can be estimated from $\mathrm{d} W / \mathrm{d} V$ in Fig. 37. The results are given in Fig. 42. 


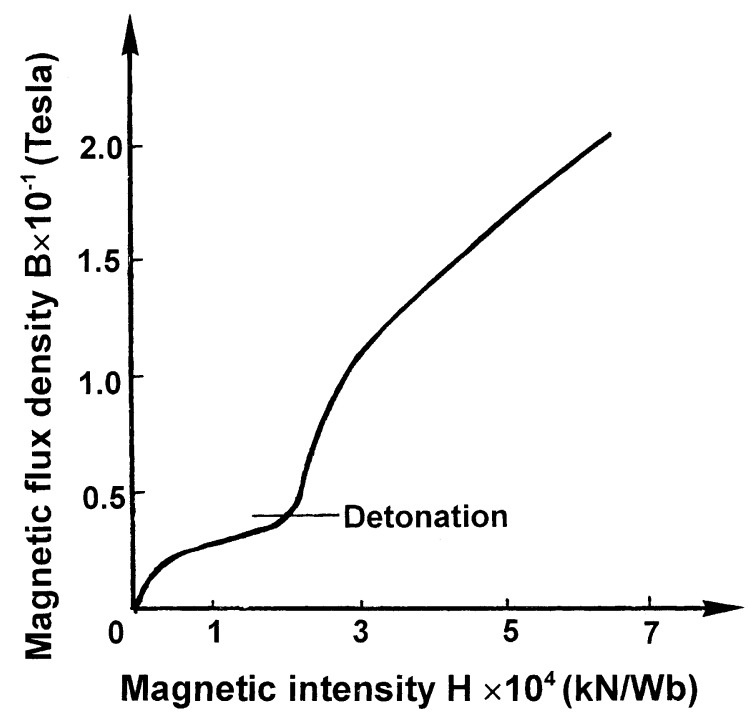

Fig. 40. Magnetic flux density versus intensity for a $70 \mathrm{~kJ}$ capacitor bank railgun system.

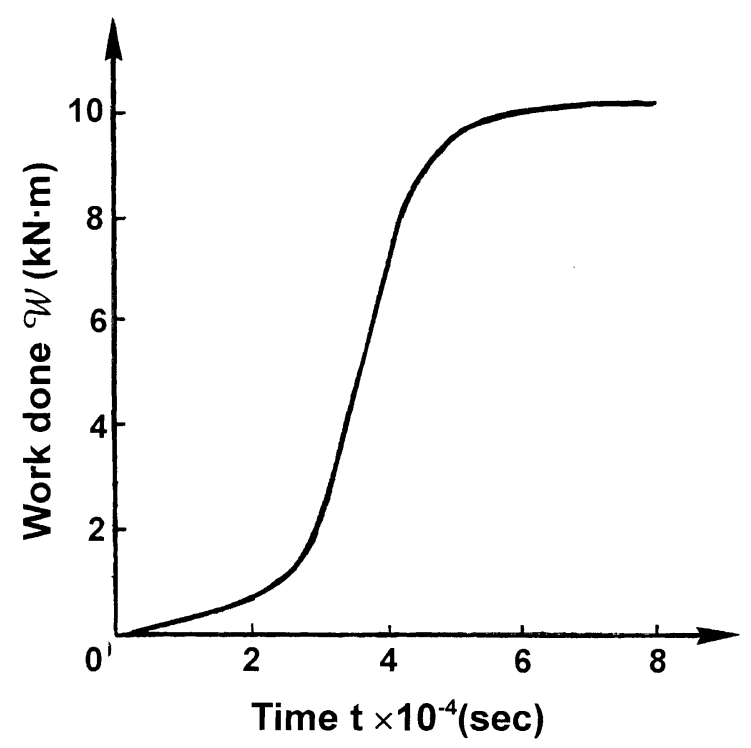

Fig. 41. Work done on projectile as a function of time for a 70 railgun system.

For the mechanical system, Eq. (1) can be put into an equivalent form

$\frac{\mathrm{d} \Theta}{\Theta}=-\frac{\mathrm{d} \sigma \mathrm{d} \epsilon}{\mathrm{d} D}$.

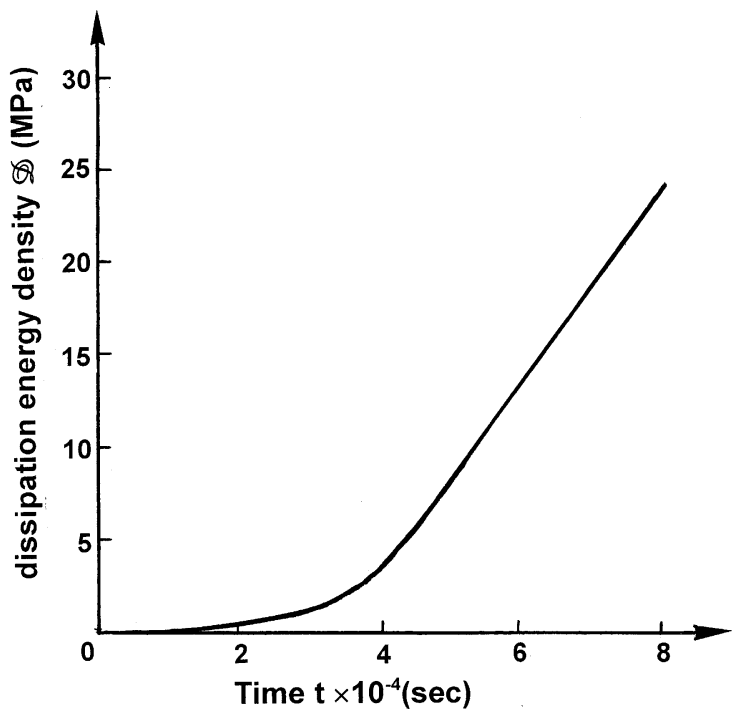

Fig. 42. Time variations of dissipation energy density for a $70 \mathrm{~kJ}$ railgun system with $15 \%$ efficiency.

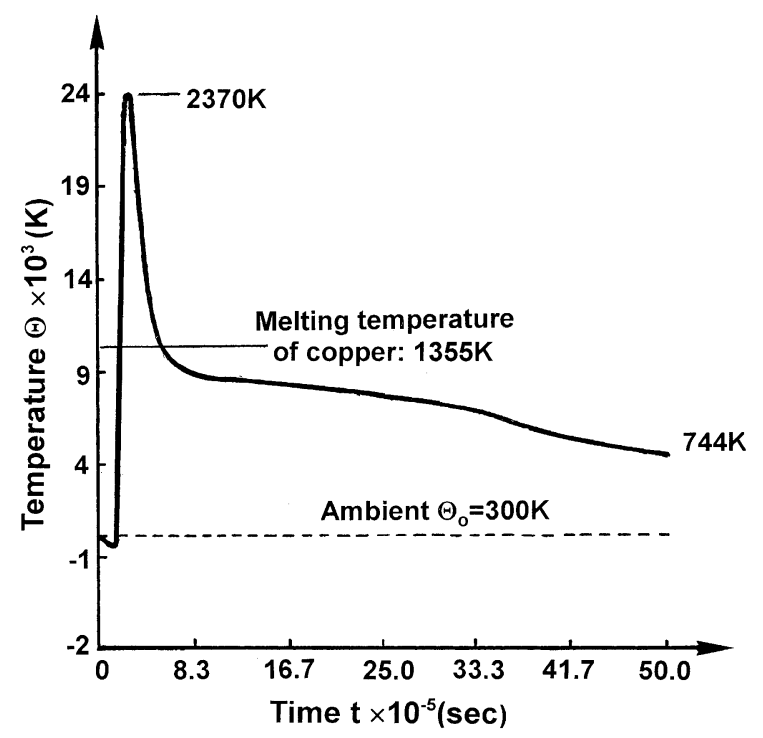

Fig. 43. Time variations of nonequilibrium temperature for a $70 \mathrm{~kJ}$ railgun system.

In Eq. (39), the induced stress $\sigma$ and induced strain $\epsilon$ are known function of time [58]. The temperature $\Theta$ can thus be solved from Eq. (39). Fig. 43 shows that the temperature rise is extremely high for the initial $2.5 \times 10^{-5} \mathrm{~s}$. This corresponds to the time 
when detonation occurred. After this initial rise, the temperature drops quickly. The peak is about $2370 \mathrm{~K}$ at $t \approx 2.5 \times 10^{-5} \mathrm{~s}$. which is beyond the melting temperature $1355 \mathrm{~K}$ for copper. But since the temperature drops very quickly, there is not sufficient time to melt large sections of the rail. Local damage, however, could take place. Of interest is the small temperature drop below ambient at $12.5 \mu \mathrm{s}$. This cooling behavior is similar to that observed for uniaxial tensile specimens. After $50 \times 10^{-5} \mathrm{~s}$, the temperature drops down to $744 \mathrm{~K}$. The rail is still very hot. The data suggest that the rail material should be made to tolerate high temperature at least for the initial $500 \mu \mathrm{s}$.

\section{Additional supporting comments}

Nanotechnology was once a laboratory curiosity; it now has the potential to revolutionalize the future of industry. The thought of lining up atom by atom to fabricate use-specific materials has profound implications on what would be expected of the material scientists and mechanists in the $21 \mathrm{st}$ century.

It was as recent as 1989 that IBM scientists used a scanning tunneling microscope to spell company's three-letter logo using xenon atoms. A similar accomplishment was achieved in the same laboratory using an array of iron atoms to spell the Chinese characters yuan-tze (in sound and not configuration) which means atom. A closer look shows that the characters are lined up by objects that may resemble tennis balls. However, they are really not spherical in shape. In this respect, does atom have a definite shape and size? According to quantum mechanics,an atom does not possess definite energy although it would presumably tend to settle to a state of minimum energy. Such a view together with the uncertainty relation have made an estimate on atom's diameter. The unit of an Angström was thus adopted. The distance $0.528 \times 10^{-8} \mathrm{~cm}$ approximately one half of an Angström was thus referred to as the Bohr radius. Since then, various packing arrangements of spherical balls of unequal axes were investigated mathematically to determine those configurations that would minimize the voids left out by the contacting spheres.

\subsection{Atomic scale}

To those who work in atomistic simulation [5-9] of physical processes, it is not unrealistic to probe on the detail features of atoms rather than just accepting the model of an array of points held together by preassumed forces. How much difference would it make if these points were spaced evenly or not? Should they be regarded as stationary or moving and how? It is, therefore, prudent to determine the conditions under which these effects are significant. A simple yes or no to a specific situation would not be satisfying. It would only promote diversification for more special cases.

Modeling of atoms may adopt an energy approach even though an excited atom may not have definite energy defined by the frequency of an amplitude. For expediency, assume that it does and that its interaction with the electromagnetic field would discharge the energy. An atom could thus adopt a lower energy state. The electromagnetic field would result in a higher state of excitation. The total energy of the system remains unchanged before and after the atom passes from a state of high to low energy. It is conceivable that each atom could be assigned with changing energy; high state, ground state and low state as influenced by the electromagnetic field and its neighboring atoms using a probabilisitic approach. Nuclei can also exist at different energy levels.

Then there are subnuclear particles with different masses where the heavier ones would disintegrate into other light particles. These changes leave little room or time for a definite physical state even when the scale size is reduced to that inside the atom. Particulates are still very much in existence but their detail features becomes less clear.

\subsection{Cosmoscale}

Interestingly enough, homogeneity and isotropy characters of nature have also been a great concern in astrophysics. As the space scale is enlarged, observations tend to favor the continuum model. 
Again, disparity between the corpuscular and the smooth continuum arises. It was in the early 1930s that recession velocities were deduced from Dopler redshifts in the spectra of nebulae outside the Milky Way. Hubble estimated these velocities to be of the order of kilometers per second and are directly proportional to the distances in megaparsecs $\left(1 \mathrm{Mpc}=3.08 \times 10^{19} \mathrm{~km}\right)$. The universe appears to be homogeneous and isotropic on the largest scale about $3000 \mathrm{Mpc}$. On slightly smaller scales up to $100 \mathrm{Mpc}$ the universe is said to be very inhomogeneous consisting of stars, galaxies and clusters of galaxies. Here again, the definition of homogeneity or inhomogeneity used depends on the resolution of the largest optical instruments at that time. The more recent data obtained from the Hubble telescope stationed in outerspace have shown that modern day estimates on the origin of the universe are off by at least one order of magnitude measured in billions of light year. Within the context of this discussion, it can be said that empirical evidence in favor of spatial uniformity on very large scales is quite strong. At least, the cosmologists today are in favor of adopting the continuum approach for deducing the nature of universe.

\subsection{Common features}

The missing mass problem in astrophysics also suggests that the vast majority of events in the universe may be predicted by elementary particle theory. There is the belief that all physical reality is subject to one set of laws. These laws may possibly be discovered with the aid of data obtained from terrestrial experiments and astronomical observations. Many spacetime models have been advanced; they are not unlike the isoenergy density theory [15] where time is coupled with space size in an implicit manner. The isoenergy density space interlaces energy change with space that could expand and contract in three directions with time. Translated into the classical sense, the spatial measuring scales in the three directions are different depending on prevailing local displacements that could arise from changes in energy regardless of the source. They could be associated with force fields in Table 4. The measurement scheme in the isoenergy density theory is analogous to a set of $x$, $y$ and $z$ axes where each could expand or contract by different amounts as if these scales were marked on a rubber sheet. If the sheet were extended differently in the three directions, then the scales would expand or contract accordingly. Such a feature accounts for local inhomogeneity. This has been reflected in many nonequilibrium thermomechanics problems that can be found in [14].

Worthwhile mentioning is that $\Delta T / T$ has been used in astrophysics as a measure of homogeneity and isotropy of cosmic microwave background radiation where $\Delta T / T<10^{-3}$ was reported to be a limit. Recall that $\mathrm{d} \Theta / \Theta$ in Eq. (1) and $\Delta T / T$ in Eq. (9) correspond to nonequilibrium and equilibrium processes, respectively, for analyzing the temperature change in stressed solids. In particular, $\mathrm{d} \Theta / \Theta$ in Eq. (1) is related directly to $\mathrm{d} V / \mathrm{d} A$ which has been used for ranking the inhomogeneity of Permalloy 65 [52] during magnetization. There is no reason why $\mathrm{d} V / \mathrm{d} A$ could not be used to study events at the cosmoscale. Another field known as "thermal cracking" [59] or pyrolysis has discovered that the volume to surface effects are critical in the cracking furnace. The term thermal cracking is defined as the thermal decomposition plus some rearrangement of hydrocarbon molecules exposed to high temperatures. The technology is concerned with the production of propylene, butadiene, aromatic compounds and hydrogen. It involves large amounts of heat transfer because pyrolysis reactions are overall highly endothermic. In laboratory furnaces with low volume-to-surface ratio, the importance of surface reactions has been appreciated for sometime. The importance of these effects in larger commercial units with much larger volume-to-surface ratio has been debated. Information accumulated recently has proved that volume-surface effects are much more important than previously anticipated.

\subsection{Closing remarks}

The advancement of science probably cannot do without the dual concepts of the particulate and smooth continuum. Nature in its true form may not be observable by scientific means. What matters is the consistent assessment of how nature 
might appear and hopefully that the results may serve some useful purposes for mankind.

\section{References}

[1] M. Chediak, R. Recinto, N. McKay, Nanotech. Grows, Red Herring 99 (2001) 47-58.

[2] A.A. Griffith, The phenomena of rupture and flow in solids, Philos. Trans. Roy. Soc. London, Series A 221 (1921) 163-198.

[3] K.J. Cheng, S.Y. Cheng, Boundary conditions of electrons at interface. Part I: Mixture of nanometer crystals and amorphous silicon, in: G.C. Sih, V.E. Panin (Eds.), Prospects of Mesomechanics in the 21st Century (special issue), J. Theoret. Appl. Fract. Mech. 37 (2001) 11-17.

[4] K.J. Cheng, S.Y. Cheng, Boundary conditions of electrons at interface. Part II: Internal stresses in thin films, in: G.C. Sih, V.E. Panin (Eds.), Prospects of Mesomechanics in the 21st Century (special issue), J. Theoret. Appl. Fract. Mech. 37 (2001) 19-27.

[5] J.B. Adams, A. Rockett, J. Kieffer, W. Xu, M. Nomura, K.A. Kilian, D.F. Richards, R. Ramprasad, Atomic-level computer simulation, J. Nucl. Mater. 216 (1994) 265-274.

[6] Proceedings of the Eurio Fermi Summer School on Molecular dynamics Simulation of Statistical Mechanics Systems, Verenna, 1985.

[7] Monte Carlo Methods in Statistical Physics, 2nd ed., Springer, Berlin, 1984.

[8] M.F. Horstemeyer, M.I. Baskes, Atomistic finite deformation simulations: a discussion on length scale effects in relation to mechanical stresses, J. Eng. Mater. Technol. 121 (1999) 114-119.

[9] M.F. Horstemeyer, M.I. Baskes, S.T. Plimpton, Atomistic simulation of size scales and strain rate effects on plasticity, J. Metals (in press).

[10] G.C. Sih, D.Y. Tzou, Heating preceded by cooling ahead of crack: macrodamage free zone, J. Theoret. Appl. Fract. Mech. 6 (2) (1986) 103-111.

[11] G.C. Sih, Thermal/mechanical interaction associated with the micromechanics of material behavior, Technical Report, Institute of Fracture and Solid Mechanics, Lehigh University, 1987.

[12] D.Y. Tzou, G.C. Sih, Thermal/mechanical interaction of subcritical crack growth in tensile specimen, J. Theoret. Appl. Fract. Mech. 10 (1) (1988) 59-72.

[13] S.M Ohr, An electron microscope study of crack tips deformation and its impact on the dislocation tiny of fracture, Mater. Sci. Eng. 72 (1985) 1-35.

[14] G.C. Sih, in: S. Sienietyez, P. Salamon (Eds.), Some Basic Problems in Nonequilibrium Thermomechanics, Taylor \& Francis, New York, 1992, pp. 218-247.

[15] G.C. Sih, Thermomechanics of solids: nonequilibrium and irreversibility, J. Theoret. Appl. Fract. Mech. 9 (3) (1988) 175-198.
[16] G.C. Sih, Experimental fracture mechanics: strain energy density critterion, in: Mech. Fract., vols. VII, XVII-LVI, Martinus Nijhoff, The Netherlands, 1981.

[17] G.C. Sih, Micromechanics associated with thermal $/$ mechanical interaction for polycrystals, in: G.C. Sih (Ed.), Mesomechanics 2000: Role of Mechanics for Development of Science and Technology, vol. 1, Tsinghua University Press, 2000, pp. 143-152.

[18] G.C. Sih, Strain energy density factor applied to mixed mode crack problems, Int. J. Fract. Mech 10 (1974) 85106.

[19] M.Z. Kipp, G.C. Sih, The strain energy density failure criterion applied to notch elastic solids, Int. J. Solids Struct. 11 (1975) 153-173.

[20] H. Noguchi, Y. Furuya, A method of seamlessly combining a crack tip molecular dynamics enclave with a linear elastic outer domain in simulating elastic-plastic crack advance, Int. J. Fract. 87 (1997) 309-329.

[21] Y. Furuya, H. Nagachi, A combined method of molecular dynamics with micromechanics improved by moving the molecular dynamics region successively in the simulation of elastic-plastic crack propagation, Int. J. Fract. 94 (1998) $17-31$.

[22] G.C. Sih, Z.F. Song, Dynamic analysis of tetragonal perovskite structure ceramics, implicated by asymptotic field solutions and boundary conditions, J. Theoret. Appl. Fract. Mech. 38 (1) (2002).

[23] G.C. Sih, B. Liu, Mesofracture mechanics: a necessary link, in: G.C. Sih, V.E. Panin (Eds.), Prospects of Mesomechanics in the 21st Century (special issue), J. Theoret. Appl. Fract. Mech. 37 (2001) 371-395.

[24] G.C. Sih, Dynamic aspects of crack propagation, in: M.P. Kanninen, W.F. Adler, A.R. Rosenfield, R.I. Jaffee (Eds.), Inelastic Behavior of Solids, McGraw-Hill, New York, 1970, pp. 607-639.

[25] R.J. Hartrant, G.C. Sih, Application of the strain energy density fracture criterion to dynamic crack problems, in: G.C. Sih, H.C. vanElst, D. Broek (Eds.), Proceedings of Prospects of Fracture Mechanics, Noordhoff, Leiden, 1973, pp. 449-458.

[26] G.C. Sih, Dynamic crack problem - strain energy density fracture theory, in: G.C. Sih (Ed.), Elastodynamic Crack Problems, Noordhoff, Leiden, 1977, pp. 17-47.

[27] G.C. Sih, C.K. Chao, Failure initiation in unnotched specimens subjected to monotonic and cyclic loading, J. Theoret. Appl. Fract. Mech. 2 (1) (1984) 67-74.

[28] G.C. Sih, A special theory of crack propagation, in: G.C. Sih (Ed.), Methods of Analysis and Solutions of Crack Problems, Noordhoff, Leiden, 1973, pp. 21-45.

[29] G.C. Sih, Fracture mechanics of engineering structural components, in: G.C. Sih, L. Faria (Eds.), Fracture Mechanics Methodology, Martinus Nijhoff, Dordrecht, 1984, pp. 35-101 (Now Kluwer Academic Publishers, Boston).

[30] G.C. Sih, Mechanics of Fracture Initiation and Propagation, Kluwer Academic Publishers, The Netherlands, 1991. 
[31] J. Wang, I-. Chin, Chinese Social Science Publishing Company, Beijing, 1990.

[32] P.W. Mast, L.A. Beaytien, M. Clifford, D.R. Mulville, S.A. Sutton, R.W. Thomas, J. Tirosh, J. Wolock, A semiautomated in-plane loader for material testing, J. Exp. Mech. 23 (2) (1983) 236-241.

[33] P.W. Mast, G.E. Nash, J. Michopoulos, R.W. Thomas, R. Badaliance, I. Wolock, Characterization of strain induced damage in composites based on the dissipated energy density - Part I: Basic scheme and formulation; Part II: Composite specimens and naval structures; and Part III: General material constitutive relation, J. Theoret. Appl. Fract. Mech. 22 (2) (1995) 71-125.

[34] G.C. Sih, D.M. Chou, Nonequilibrium thermal/mechanical response 6061 of aluminum alloy at elevated temperature, J. Theoret. Appl. Fract. Mech. 12 (1) (1989) 19-31.

[35] G.C. Sih, D.Y. Tzou, J.G. Michopoulos, Secondary temperature fluctuation in cracked 1020 steel specimen loaded monotonically, J. Theoret. Appl. Fract. Mech. 7 (2) (1987) 79-89.

[36] G.C. Sih, D.Y. Tzou, Irreversibility and damage of SAFC40R steel specimen in uniaxial tension, J. Theoret. Appl. Fract. Mech. 7 (1) (1987) 23-30.

[37] G.C. Sih, F.L. Lieu, C.K. Chao, Thermal/mechanical damage of 6061-T6 aluminum tensile specimen, J. Theoret. Appl. Fract. Mech. 7 (2) (1987) 67-78.

[38] G.C. Sih, D.H. Song, Time dependent damage in half plane - Part I: displacement loading, J. Theoret. Appl. Fract. Mech. 9 (3) (1988) 199-215.

[39] G.C. Sih, D.H. Song, Time dependent damage in half plane - Part II: traction loading, J. Theoret. Appl. Fract. Mech. 9 (3) (1988) 199-215.

[40] G.C. Sih, C.K. Chao, Scaling of size/time/temperature associated with damage of uniaxial specimens - Part I: Progressive damage in uniaxial tensile specimen, J. Theoret. Appl. Fract. Mech. 12 (2) (1989) 93-108.

[41] G.C. Sih, C.K. Chao, Scaling of size/time/temperature associated with damage of uniaxial specimens - Part II: Progressive damage in uniaxial compressive specimen, J. Theoret. Appl. Fract. Mech. 12 (2) (1989) 109119.

[42] G.C. Sih, Initial damage states of hypervelocity impact for tungsten projectile, J. Theoret. Appl. Fract. Mech. 13 (1990) 167-180.

[43] B.L. Holian, S.J. Zhou, P.S. Lomdahl, N. GronbechJensen, D.M. Beazley, R. Ravelo, Molecular-dynamics simulation of fracture: an overview of system size and other effects, Mater. Res. Soc. Symp. Proc. 409 (1996) 3-9.

[44] Yu, Sklyadneva, G.G. Rusina, E.V. Chulkov, Vibrational states in vicinal surfaces of Al, Ag, Cu, Pd, J. Surf. Sci. 416 (1998) 17-36.
[45] Yu, Sklyadneva, G.G. Rusina, E.V. Chulkov, Vibrations on $\mathrm{Cu}$ surface covered with Ni monolayer, J. Surf. Sci. 435 (1999) 517-519.

[46] W. Thompson Sir, in: Mathematical and Physical Papers, vol. 3, Cambridge University Press, London, 1890, pp. 6382; W. Thompson Sir, On thermo-elastic and thermomagnetic properties of matter, Quarterly J. Math. 1 (1857) 57-77.

[47] G.C. Sih, The role of surface and volume energy in the mechanisms of fracture, in: V. Balakrishnan, C.E. Bottani (Eds.), Mechanical Properties and Behavior of Solids: Plastic Instability, World Scientific, Singapore, 1985, pp. 396-461.

[48] G.C. Sih, Strain energy density and surface layer energy for blunt cracks or notches, in: G.C. Sih (Ed.), Stress Analysis of Notch Problems, Noordhoff, Leiden, 1978, pp. 13-110.

[49] P.M. Nadai, Untersuchungen der Festigkeitslehre mit Hilfe von Temperature-messungen, Dissertation, Technische Hochschule, Berlin, 1911.

[50] G.C. Sih, Reconciliation of surface and volume energy density in continuum mechanics, in: M.H. Yu, S.C. Fan (Eds.), Strength Theory, Science Press, Beijing, 1998, pp. $67-78$.

[51] G.C. Sih, Thermal/mechanical penetration damage of 4340 steel impacted by tungsten projectile, in: E.P. Chen, V.R. Luk (Eds.), Advances in Numerical Simulation Technique for Penetration and Perforation of Solids, ASME Appl. Mech. Div. 171 (1993) 133-145.

[52] Y.D. Lee, Nonequilibrium thermoelectromagnetic response in ferromagnetic solids, Ph.D. Thesis, Lehigh University, Bethlehem, PA, 1993.

[53] O. Horger (Ed.), ASME Handbook on Metals Engineering Design, second ed., 1965.

[54] R.M. Bozorth, Ferromagnetism, D.V. Nostrand Company Inc., New York, 1951.

[55] G.C. Sih, Thermal/mechanical Interaction Associated with the Micromechanics of Material Behavior, Lehigh University Institute of Fracture and Solid Mechanics Publication, 1987.

[56] R.S. Hawke, A.L. Brooks, F.J. Deadrick, J.K. Scudder, Results of railgun experiments powered by magnetic flux compression generators, IEEE Trans. Mag. Mag-18 (1981).

[57] F.J. Deadrick, R.S. Hawke, J.K. Scudder, MAGRAC - a railgun simulation program, Conference on Electromagnetic Guns and Launchers, 1980.

[58] G.C. Sih, Design and development of electromagnetic launcher device, Technical Report, Institute of Fracture and Solid Mechanics, Lehigh University, Bethlehem, PA, 1993.

[59] B.L. Crymes, L.F. Albright, in: Cracking, Thermal, Encyclopedia of Physical Science and Technology, vol. 3, Academic Press, New York, 1987, pp. 768-785. 